\title{
JOHN VON NEUMANN
}

\section{3-1957}

\section{S. ULAM}

In John von Neumann's death on February 8, 1957, the world of mathematics lost a most original, penetrating, and versatile mind. Science suffered the loss of a universal intellect and a unique interpreter of mathematics, who could bring the latest (and develop latent) applications of its methods to bear on problems of physics, astronomy, biology, and the new technology. Many eminent voices have already described and praised his contributions. It is my aim to add here a brief account of his life and of his work from a background of personal acquaintance and friendship extending over a period of 25 years.

John von Neumann (Johnny, as he was universally known in this country), the eldest of three boys, was born on December 28, 1903, in Budapest, Hungary, at that time part of the Austro-Hungarian empire. His family was well-to-do; his father, Max von Neumann, was a banker. As a small child, he was educated privately. In 1914, at the outbreak of the First World War, he was ten years old and entered the gymnasium.

Budapest, in the period of the two decades around the First World War, proved to be an exceptionally fertile breeding ground for scientific talent. It will be left to historians of science to discover and explain the conditions which catalyzed the emergence of so many brilliant individuals (-their names abound in the annals of mathematics and physics of the present time). Johnny was probably the most brilliant star in this constellation of scientists. When asked about his own opinion on what contributed to this statistically unlikely phenomenon, he would say that it was a coincidence of some cultural factors which he could not make precise: an external pressure on the whole society of this part of Central Europe, a subconscious feeling of extreme insecurity in individuals, and the necessity of producing the unusual or facing extinction. The First World War had shattered the existing economic and social patterns. Budapest, formerly the second capital of the Austro-Hungarian empire, was now the principal town of a small country. It became obvious to

Received by the editors February 8, 1958. 
many scientists that they would have to emigrate and find a living elsewhere in less restricted and provincial surroundings.

According to Fellner, ${ }^{1}$ who was a classmate of his, Johnny's unusual abilities came to the attention of an early teacher (Laslo Ratz). He expressed to Johnny's father the opinion that it would be nonsensical to teach Johnny school mathematics in the conventional way, and they agreed that he should be privately coached in mathematics. Thus, under the guidance of Professor Kürschak and the tutoring of Fekete, then an assistant at the University of Budapest, he learned about the problems of mathematics. When he passed his "matura" in 1921, he was already recognized a professional mathematician. His first paper, a note with Fekete, was composed while he was not yet 18. During the next four years, Johnny was registered at the University of Budapest as a student of mathematics, but he spent most of his time in Zurich at the Eidgenössische Technische Hochschule, where he obtained an undergraduate degree of "Diplomingenieur in Chemie," and in Berlin. He would appear at the end of each semester at the University of Budapest to pass his course examinations (without having attended the courses, which was somewhat irregular). He received his doctorate in mathematics in Budapest at about the same time as his chemistry degree in Zurich. While in Zurich, he spent much of his spare time working on mathematical problems, writing for publication, and corresponding with mathematicians. $\mathrm{He}$ had contacts with Weyl and Polya, both of whom were in Zurich. At one time, Weyl left Zurich for a short period, and Johnny took over his course for that period.

It should be noted that, on the whole, precocity in original mathematical work was not uncommon in Europe. Compared to the United States, there seems to be a difference of at least two or three years in specialized education, due perhaps to a more intensive schooling system during the gymnasium and college years. However, Johnny was exceptional even among the youthful prodigies. His original work began even in his student days, and in 1927, he became a Privat Dozent at the University of Berlin. He held this position for three years until 1929, and during that time, became well-known to the mathematicians of the world through his publications in set theory, algebra, and quantum theory. I remember that in 1927, when he came to Lwów (in Poland) to attend a congress of mathematicians, his work in foundations of mathematics and set theory was already famous. This was already mentioned to us, a group of students, as an example of the work of a youthful genius.

1 This information was communicated by Fellner in a letter recalling Johnny's early studies. 
In 1929, he transferred to the University of Hamburg, also as a Privat-Dozent, and in 1930, he came to this country for the first time as a visiting lecturer at Princeton University. I remember Johnny telling me that even though the number of existing and prospective vacancies in German universities was extremely small, most of the two or three score Dozents counted on a professorship in the near future. With his typically rational approach, Johnny computed that the expected number of professorial appointments within three years was three, the number of Dozents was 40 ! He also felt that the coming political events would make intellectual work very difficult.

He accepted a visiting professorship at Princeton in 1930, lecturing for part of the academic year and returning to Europe in the summers. He became a permanent professor at the University in 1931 and held this position until 1933 when he was invited to join the Institute for Advanced Study as a professor, the youngest member of its permanent faculty.

Johnny married Marietta Kovesi in 1930. His daughter, Marina, was born in Princeton in 1935. In the early years of the Institute, a visitor from Europe found a wonderfully informal and yet intense scientific atmosphere. The Institute professors had their offices at Fine Hall (part of Princeton University), and in the Institute and the University departments a galaxy of celebrities was included in what quite possibly constituted one of the greatest concentrations of brains in mathematics and physics at any time and place.

It was upon Johnny's invitation that I visited this country for the first time at the end of 1935. Professor Veblen and his wife were responsible for the pleasant social atmosphere, and I found that the von Neumann's (and Alexander's) houses were the scenes of almost constant gatherings. These were the years of the depression, but the Institute managed to give to a considerable number of both native and visiting mathematicians a relatively carefree existence.

Johnny's first marriage terminated in divorce. In 1938, he remarried during a summer visit to Budapest and brought back to Princeton his second wife, Klara Dan. His home continued to be a gathering place for scientists. His friends will remember the inexhaustible hospitality and the atmosphere of intelligence and wit one found there. Klari von Neumann later became one of the first coders of mathematical problems for electronic computing machines, an art to which she brought some of its early skills.

With the beginning of the war in Europe, Johnny's activities outside the Institute started to multiply. A list of his positions, organizational memberships, etc., will be found at the end of this article. This mere enumeration gives an idea of the enormous amount of work 
Johnny was performing for various scientific projects in and out of the government.

In October, 1954, he was named by presidential appointment as a member of the United States Atomic Energy Commission. He left Princeton on a leave of absence and discontinued all commitments with the exception of the chairmanship of the ICBM Committee. Admiral Strauss, chairman of the Commission and a friend of Johnny's for many years, suggested this nomination as soon as a vacancy occurred. Of Johnny's brief period of active service on the Commission, he writes:

"During the period between the date of his confirmation and the late autumn, 1955, Johnny functioned magnificently. He had the invaluable faculty of being able to take the most difficult problem, separate it into its components, whereupon everything looked brilliantly simple, and all of us wondered why we had not been able to see through to the answer as clearly as it was possible for him to do. In this way, he enormously facilitated the work of the Atomic Energy Commission."

Johnny, whose health had always been excellent, began to look very fatigued in 1954. In the summer of 1955 , the first symptoms of a fatal disease were discovered by $\mathrm{x}$-ray examination. A prolonged and cruel illness gradually put an end to all his activities. He died at Walter Reed Hospital in Washington at the age of 53.

Johnny's friends remember him in his characteristic poses: standing before a blackboard or discussing problems at home. Somehow, his gesture, smile, and the expression of the eyes always reflected the kind of thought or the nature of the problem under discussion. He was of middle size, quite slim as a very young man, then increasingly corpulent; moving about in small steps with considerable random acceleration, but never with great speed. A smile flashed on his face whenever a problem exhibited features of a logical or mathematical paradox. Quite independently of his liking for abstract wit, he had a strong appreciation (one might say almost a hunger) for the more earthy type of comedy and humor.

He seemed to combine in his mind several abilities which, if not contradictory, at least seem separately to require such powers of concentration and memory that one very rarely finds them together in one intellect. These are: a feeling for the set-theoretical, formally algebraic basis of mathematical thought, the knowledge and understanding of the substance of classical mathematics in analysis and geometry, and a very acute perception of the potentialities of modern mathematical methods for the formulation of existing and new problems of theoretical physics. All this is specifically demonstrated by 
his brilliant and original work which covers a very wide spectrum of contemporary scientific thought.

His conversations with friends on scientific subjects could last for hours. There never was a lack of subjects, even when one departed from mathematical topics.

Johnny had a vivid interest in people and delighted in gossip. One often had the feeling that in his memory he was making a collection of human peculiarities as if preparing a statistical study. He followed also the changes brought by the passage of time. When a young man, he mentioned to me several times his belief that the primary mathematical powers decline after the age of about 26 , but that a certain more prosaic shrewdness developed by experience manages to compensate for this gradual loss, at least for a time. Later, this limiting age was slowly raised.

He engaged occasionally in conversational evaluations of other scientists; he was, on the whole, quite generous in his opinions, but often able to damn by faint praise. The expressed judgment was, in general, very cautious, and he was certainly unwilling to state any final opinions about others: "Let Rhadamantys and Minos... judge ...." Once when asked, he said that he would consider Erhard Schmidt and Hermann Weyl among the mathematicians who especially influenced him technically in his early life.

Johnny was regarded by many as an excellent chairman of committees (this peculiar contemporary activity). He would press strongly his technical views, but defer rather easily on personal or organizational matters.

In spite of his great powers and his full consciousness of them, he lacked a certain self-confidence, admiring greatly a few mathematicians and physicists who possessed qualities which he did not believe he himself had in the highest possible degree. The qualities which evoked this feeling on his part were, I felt, relatively simple-minded powers of intuition of new truths, or the gift for a seemingly irrational perception of proofs or formulation of new theorems.

Quite aware that the criteria of value in mathematical work are, to some extent, purely aesthetic, he once expressed an apprehension that the values put on abstract scientific achievement in our present civilization might diminish: "The interests of humanity may change, the present curiosities in science may cease, and entirely different things may occupy the human mind in the future." One conversation centered on the ever accelerating progress of technology and changes in the mode of human life, which gives the appearance of approaching some essential singularity in the history of the race beyond which human affairs, as we know them, could not continue. 
His friends enjoyed his great sense of humor. Among fellow scientists, he could make illuminating, often ironical, comments on historical or social phenomena with a mathematician's formulation, exhibiting the humor inherent in some statement true only in the vacuous set. These often could be appreciated only by mathematicians. He certainly did not consider mathematics sacrosanct. I remember a discussion in Los Alamos, in connection with some physical problems where a mathematical argument used the existence of ergodic transformations and fixed points. He remarked with a sudden smile, "Modern mathematics can be applied after all! It isn't clear, a priori, is it, that it could be so . . .."

I would say that his main interest after science was in the study of history. His knowledge of ancient history was unbelievably detailed. He remembered, for instance, all the anecdotical material in Gibbon's Decline and Fall and liked to engage after dinner in historical discussions. On a trip south, to a meeting of the American Mathematical Society at Duke University, passing near the battlefields of the Civil War he amazed us by his familiarity with the minutest features of the battles. This encyclopedic knowledge molded his views on the course of future events by inducing a sort of analytic continuation. I can testify that in his forecasts of political events leading to the Second World War and of military events during the war, most of his guesses were amazingly correct. After the end of the Second World War, however, his apprehensions of an almost immediate subsequent calamity, which he considered as extremely likely, proved fortunately wrong. There was perhaps an inclination to take a too exclusively rational point of view about the cases of historical events. This tendency was possibly due to an over-formalized game theory approach.

Among other accomplishments, Johnny was an excellent linguist. He remembered his school Latin and Greek remarkably well. In addition to English, he spoke German and French fluently. His lectures in this country were well known for their literary quality (with very few characteristic mispronunciations which his friends anticipated joyfully, e.g., "integhers"). During his frequent visits to Los Alamos and Santa Fe (New Mexico), he displayed a less perfect knowledge of Spanish, and on a trip to Mexico, he tried to make himself understood by using "neo-Castilian," a creation of his own -English words with an "el" prefix and appropriate Spanish endings.

Before the war, Johnny spent the summers in Europe on vacations and lecturing (in 1935 at Cambridge University, in 1936 at the Institut Henri Poincaré in Paris). Often he mentioned that per- 
sonally he found doing scientific work there almost impossil!e because of the atmosphere of political tension. After the war he undertook trips abroad only unwillingly.

Ever since he came to the United States, he expressed his appreciation of the opportunities here and very high hopes for the future of scientific work in this country.

To follow chronologically von Neumann's interests and accomplishments is to review a large part of the whole scientific development of the last three decades. In his youthful work, he was concerned not only with mathematical logic and the axiomatics of set theory, but, simultaneously, with the substance of set theory itself, obtaining interesting results in measure theory and the theory of real variables. It was in this period also that he began his classical work on quantum theory, the mathematical foundation of the theory of measurement in quantum theory and the new statistical mechanics. His profound studies of operators in Hilbert spaces also date from this period. He pushed far beyond the immediate needs of physical theories, and initiated a detailed study of rings of operators, which has independent mathematical interest. The beginning of the work on continuous geometries belongs to this period as well.

Von Neumann's awareness of results obtained by other mathematicians and the inherent possibilities which they offer is astonishing. Early in his work, a paper by Borel on the minimax property led him to develop in the paper, Zur Theorie der Gesellschaft-Spiele, ${ }^{2}$ ideas which culminated later in one of his most original creations, the theory of games. An idea of Koopman on the possibilities of treating problems of classical mechanics by means of operators on a function space stimulated him to give the first mathematically rigorous proof of an ergodic theorem. Haar's construction of measure in groups provided the inspiration for his wonderful partial solution of Hilbert's fifth problem, in which he proved the possibility of introducing analytical parameters in compact groups.

In the middle 30's, Johnny was fascinated by the problem of hydrodynamical turbulence. It was then that he became aware of the mysteries underlying the subject of non-linear partial differential equations. His work, from the beginning of the Second World War, concerns a study of the equations of hydrodynamics and the theory of shocks. The phenomena described by these non-linear equations are baffling analytically and defy even qualitative insight by present

\footnotetext{
2 Paper [17].
} 
methods. Numerical work seemed to him the most promising way to obtain a feeling for the behavior of such systems. This impelled him to study new possibilities of computation on electronic machines, $\mathrm{ab}$ initio. He began to work on the theory of computing and planned the work, to remain unfinished, on the theory of automata. It was at the outset of such studies that his interest in the working of the nervous system and the schematized properties of organisms claimed so much of his attention.

This journey through many fields of mathematical sciences was not a result of restlessness. Neither was it a search for novelty, nor a desire for applying a small set of general methods to many diverse special cases. Mathematics, in contrast to theoretical physics, is not confined to a few central problems. The search for unity, if pursued on a purely formal basis, von Neumann considered doomed to failure. This wide range of curiosity had its basis in some metamathematical motivations and was influenced strongly by the world of physical phenomena - these will probably defy formalization for a long time tocome.

Mathematicians, at the outset of their creative work, are often confronted by two conflicting motivations: the first is to contribute to the edifice of existing work-it is there that one can be sure of gaining recognition quickly by solving outstanding problems-the second is the desire to blaze new trails and to create new syntheses. This latter course is a more risky undertaking, the final judgment of value or success appearing only in the future. In his early work, Johnny chose the first of these alternatives. It was toward the end of his life that he felt sure enough of himself to engage freely and yet painstakingly in the creation of a possible new mathematical discipline. This was to be a combinatorial theory of automata and organisms. His illness and premature death permitted him to make only a beginning.

In his constant search for applicability and in his general mathematical instinct for all exact sciences, he brought to mind Euler, Poincaré, or in more recent times, perhaps Hermann Weyl. One should remember that the diversity and complexity of contemporary problems surpass enormously the situation confronting the first two named. In one of his last articles, Johnny deplored the fact that it does not seem possible nowadays for any one brain to have more than a passing knowledge of more than one-third of the field of pure mathematics.

Early work, set-theory, algebra. The first paper, a joint work with Fekete, deals with zeros of certain minimal polynomials. It concerns 
a generalization of Fejér's theorem on location of the roots of Tchebyscheff polynomials. Its date is 1922 . Von Neumann was not quite eighteen when the article appeared.

Another youthful work is contained in a paper (in Hungarian with a German summary) on uniformly dense sequences of numbers. It contains a theorem on the possibility of re-ordering dense sequences of points so they will become uniformly dense; the work does not yet indicate the future depth of formulations nor is it technically difficult, but the choice of subject and the conciseness of technique in proofs begins to indicate the combination of set-theoretical intuition and the algebraic technique of his future investigations.

The set-theoretical orientation in the thinking of a great number of young mathematicians is quite characteristic of this era. The great ideas of George Cantor, which found their fruition finally in the theory of real variables, topology and later in analysis, through the work of the great Frenchmen, Baire, Borel, Lebesgue, and others, were not yet commonly part of the fundamental intuitions of young mathematicians at the turn of the century. After the end of the First World War, however, one notices that these ideas became more, as it were, naturally instinctive for the new generation.

Paper [2] in the Acta Szeged on transfinite ordinals already shows von Neumann in his characteristic form and style in dealing with the algebraic treatment of set theory. The first sentence states frankly: "The aim of this work is to formulate concretely and precisely the idea of Cantor's ordinal numbers." As the preface states, the heretofore somewhat vague formulation of Cantor himself is replaced by definitions which can be given in the system of axioms of Zermelo. Moreover, a rigorous foundation for the definition by transfinite induction is outlined. The introduction stresses the strictly formalistic approach, and von Neumann states somewhat proudly that the symbols... (for "et cetera") and similar expressions are never employed. This treatment of ordinal numbers, later also considered by Kuratowski, is to this day the best introduction of this idea, so important for "constructions" in abstract set theory. Each ordinal number by von Neumann's definition is the set of all smaller ordinal numbers. This leads to a most elegant theory and moreover allows one to avoid the concept of ordertype, which is vague insofar as the set of all ordered sets similar to a given one does not exist in axiomatic set theory.

Paper [5] on Prüfer's theory of ideal algebraic numbers begins to indicate his future breadth of interests. The paper deals with set theoretical questions and enumeration problems about relatively 
prime ideal components. Prüfer had introduced ideal numbers as "ideal solutions of infinite systems of congruences." Von Neumann starts with methods analogous to Kürschak and Bauer's work on Hensel's $p$-adic numbers. Here again, von Neumann exhibits the techniques which were to become so prevalent in the following decades in mathematical research-of continuing algebraical constructions, originally considered on finite sets, to the domain of the infinitely enumerable and the continuum. Another indication of his algebraic interests is a short note [39] on Minkowski's theory of linear forms.

A desire to axiomatize - and this in a sense more formal and precise than that originally considered by logicians at the beginning of the 20 th century-shows through much of the early work. From around 1925 to 1929 , most of von Neumann's papers deal with attempts to spread the spirit of axiomatization even through physical theory. Not satisfied with the existing formulations, even in set theory itself, he states again quite frankly in the first sentence of his paper [3] on the axiomatization of set theory:3 "The aim of the present work is to give a logically unobjectionable axiomatic treatment of set theory"; the next sentence reads, "I would like to say something at first about difficulties which make such a construction of set theory desirable."

The last sentence of this 1925 paper is most interesting. Von Neumann points out the limits of any axiomatic formulation. There is here perhaps a vague forecast of Gödel's results on the existence of undecidable propositions in any formal system. The concluding sentence is: "We cannot, for the present, do more than to state that

${ }^{3}$ About this paper, Professor Fraenkel of the Hebrew University in Jerusalem wrote me the following:

"Around 1922-23, being then professor at Marburg University, I received from Professor Erhard Schmidt, Berlin (on behalf of the Redaktion of the Mathematische Zeitschrift) a long manuscript of an author unknown to me, Johann von Neumann, with the title Die Axiomatisierung der Mengenlehre, this being his eventual doctor dissertation which appeared in the Zeitschrift only in 1928, (Vol. 27). I was asked to express my view since it seemed incomprehensible. I don't maintain that I understood everything, but enough to see that this was an outstanding work and to recognize ex ungue leonem. While answering in this sense, I invited the young scholar to visit me (in Marburg) and discussed things with him, strongly advising him to prepare the ground for the understanding of so technical an essay by a more informal essay which should stress the new access to the problem and its fundamental consequences. He wrote such an essay under the title, Eine Axiomatisierung der Mengenlehre, and I published it in 1925 in the Journal für Mathematik (vol. 154) of which I was then Associate Editor." 
there are here objections against set theory itself, and there is no way known at present to avoid these difficulties." (One is reminded here, perhaps, of an analogous statement in an entirely different domain of science: Pauli's evaluation of the state of relativistic quantum theory written in his Handbuch der Physik article and the still mysterious role of infinities and divergences in field theory.)

His second paper [18] on this subject has the title, The axiomatization of set theory (An axiomatization of set theory was the 1925 title).

The conciseness of the system of axioms is surprising, the introduction of objects of the first and second type corresponding, respectively, to sets and properties of sets in the naive set theory; the axioms take only a little more than one page of print. This is sufficient to build up practically all of the naive set theory and therewith all of modern mathematics and constitutes, to this day, one of the best foundations for set-theoretical mathematics. Gödel, in his great work on the independence of the axiom of choice, and on the continuum hypothesis, uses a system inspired by this treatment. It is noteworthy that in his first paper on the axiomatization of set theory, von Neumann recognizes explicitly the two fundamentally different directions taken by mathematicians in order to avoid the antinomies of BuraliForti, Richard and Russell. One group, containing Russell, J. König, Brouwer, and Weyl, takes the more radical point of view that the entire logical foundations of exact sciences have to be restricted in order to prevent the appearance of paradoxes of the above type. Von Neumann says, "the general impression of their activity is almost crushing." He objects to Russell's building the system of mathematics on the highly problematic axiom of reducibility, and objects to Weyl's and Brouwer's rejection of what he considers as the greater part of mathematics and set theory.

He has more sympathy with the second less radical group, naming in it Zermelo, Fraenkel, and Schoenflies. He considers their work, including his own, as far from complete, stating explicitly that the axioms appear somewhat arbitrary. He states that one cannot show in this fashion that antinomies are really excluded but while naive set theory cannot be considered too seriously, at least much of what it contains can be rehabilitated as a formal system, and the sense of "formalistic" can be defined in a clear fashion.

Von Neumann's system gives the first foundation of set theory on the basis of a finite number of axioms of the same simple logical structure as have, e.g., the axioms of elementary geometry. The conciseness of the system of axioms and the formal character of the reasoning employed seem to realize Hilbert's goal of treating mathe- 
matics as a finite game. Here one can divine the germ of von Neumann's future interest in computing machines and the "mechanization" of proofs.

Starting with the axioms, the efficiency of the algebraic manipulation in the derivation of most of the important notions of set theory is astounding; the economy of the treatment seems to indicate a more fundamental interest in brevity than in virtuosity for its own sake. It thereby helped prepare the grounds for an investigation of the limits of finite formalism by means of the concept of "machine" or "automaton."

It seems curious to me that in the many mathematical conversations on topics belonging to set theory and allied fields, von Neumann even seemed to think formally. Most mathematicians, when discussing problems in these fields, seemingly have an intuitive framework based on geometrical or almost tactile pictures of abstract sets, transformations, etc. Von Neumann gave the impression of operating sequentially by purely formal deductions. What I mean to say is that the basis of his intuition, which could produce new theorems and proofs just as well as the "naive" intuition, seemed to be of a type that is much rarer. If one has to divide mathematicians, as Poincaré proposed, into two types-those with visual and those with auditory intuition-Johnny perhaps belonged to the latter. In him, the "auditory sense," however, probably was very abstract. It involved, rather, a complementarity between the formal appearance of a collection of symbols and the game played with them on the one hand, and an interpretation of their meanings on the other. The foregoing distinction is somewhat like that between a mental picture of the physical chess board and a mental picture of a sequence of moves on it, written down in algebraic notation.

In conversations, some quite recent, on the present status of foundations of mathematics, von Neumann seemed to imply that in his view, the story is far from having been told. Gödel's discovery should lead to a new approach to the understanding of the role of formalism in mathematics, rather than be considered as closing the subject.

Paper [16] translates into strictly axiomatic treatment what was done informally in paper [2]. The first part of the paper deals with the introduction of the fundamental operations in set theory, the foundation of the theories of equivalence, similarity, well-ordering, and finally, a proof of the possibility of definition by finite or trans-

4 This was, of course, very much in Leibniz's mind. 
finite induction, including a treatment of ordinal numbers. Von Neumann rightly insists at the end of his introduction to the paper that transfinite induction was not rigorously introduced before in any axiomatic or non-axiomatic system of set theory.

Perhaps the most interesting of von Neumann's papers on axiomatics of set theory is [23]. It has to do with a certain necessary and sufficient condition which a property of sets must satisfy in order to define a set of sets. The condition is that there must not exist a oneto-one correspondence between all sets and the sets which have the property in question. This existential principle for sets had been assumed as an axiom ${ }^{5}$ by von Neumann and some of the axioms assumed in other systems, in particular the axiom of choice, had been derived from it. Now it is shown that, vice versa, these other axioms imply von Neumann's axiom, which thereby is proved consistent, provided the usual axioms are.

No. [12] his great paper in the Mathematische Zeitschrift, Zur Hilbertschen Berweistheorie, is devoted to the problem of the freedom from contradiction of mathematics. This classical study contains an exposition of the primitive ideas underlying mathematical formalisms in general. It is stressed that the whole complex of problems, originated and developed by Hilbert and also treated by Bernays and Ackermann, have not been satisfactorily solved. In particular, it is pointed out that Ackermann's proof of freedom from contradiction cannot be carried through for classical analysis. It is replaced by a rigorous finitary proof for a certain subsystem. In fact von Neumann's proof shows (although this is not stated explicitly) that finitely iterated application of quantifiers and propositional connectives to any finitary (i.e., decidable) relations is consistent. This is not far from the limit of what can be obtained on the basis of Hilbert's original program, i.e., with strictly finitary methods. But von Neumann at that time conjectured that all of analysis can be proved consistent with the same method. At the present time, one cannot escape the impression that the ideas initiated by the work of Hilbert and his school, developed with such precision, and then revolutionized by

5 Gödel says about this axiom: "The great interest which this axiom has lies in the fact that it is a maximum principle, somewhat similar to Hilbert's axiom of completeness in geometry. For, roughly speaking, it says that any set which does not, in a certain well defined way, imply an inconsistency exists. Its being a maximum principle also explains the fact that this axiom implies the axiom of choice. I believe that the basic problems of abstract set theory, such as Cantor's continuum problem, will be solved satisfactorily only with the help of stronger axioms of this kind, which in a sense are opposite or complementary to the constructivistic interpretation of mathematics." 
Gödel, are not yet exhausted. It might be that we are in the midst of another great evolution: the "naive" treatment of set theory and the formal metamathematical attempts to contain the set of our intuitions about infinity are, I think, turning toward a future "super set theory." Several times in the history of mathematics, the intuitions or, one might better say, common vague feelings of leading mathematicians about problems of existing science, later became incorporated in a formal "super system" dealing with the essence of problems in the original system.

Von Neumann pursued his interest in problems of foundations of mathematics until the end of his life. A quarter of a century after the appearance of the above series of papers, one can see the imprint of this work in his discoveries in the plans for the logic of computing machines.

Parallel to the work on foundations of mathematics, there come specific results in set theory itself and set-theoretically motivated theorems in real variables and in algebra. For example, von Neumann shows the existence of a set $M$ of real numbers, of the power of the continuum, such that any finite number of the elements of $M$ are algebraically independent. The proof is given effectively without the axiom of choice. In a paper in Fundamenta Mathematicae, [14] the same year, a decomposition of the interval is given into countably many disjoint and congruent subsets. This solved a problem of Steinhaus-a special ingenuity is required to have such a decomposition on an interval-the corresponding construction of Hausdorff for the circumference of a circle is much easier. (This is due to the fact that the circumference of a circle may be regarded as a group manifold.)

In paper [28] on the general measure theory, in Fundamenta (1928), the problem of a finitely additive measure is treated for subsets of groups. The paradoxical decompositions of the sphere by Hausdorff and the wonderfully strange decompositions of Banach and Tarski are generalized from the Euclidean space to general nonAbelian groups. The affirmative results of Banach on the possibility of a measure for all subsets of the plane are generalized to the case of subsets of a commutative group. The final conclusion is that all solvable groups are "measurable" (i.e. such measure can be introduced in them).

The problems and methods of this article form one of the first instances of a trend which developed strongly since that time, that of generalizing the set-theoretical results from Euclidean space to more general topological and algebraic structures. The "congruence" of two sets is understood to mean equivalence under a transformation 
belonging to a given group of transformations. The measure is a general additive set function. Again, the formulation of the problem presages the work of Haar and the study of Hausdorff-BanachTarski paradoxical decompositions. ${ }^{6}$

In the same "annus mirabilis," 1928, there appears the article on the theory of games. This is his first work on what was to become later an important combinatorial theory with so many applications and developments vigorously continuing at the present time. It is hard to believe that beginning with 1927 , simultaneously with the work discussed above, he could have published numerous papers on the mathematical foundations of quantum theory, probability in statistical quantum theory, and some important results on representation of continuous groups!

Theory of functions of real variables, measure theory, topology, continuous groups. Professor Halmos' article describes von Neumann's important contributions to measure theory. We shall briefly mention some of his results in this field viewed against the background of his other work.

Paper [35] solves a problem of Haar. It concerns the selection of representatives from classes of functions which are equivalent up to a set of measure zero from linear manifolds over products of powers of finite systems. The problem is generalized to measures other than Lebesgue's and an analogous problem is solved affirmatively.

[45] contains a proof of an important fact in measure theory: Any Boolean mapping between two classes of measurable sets (on two measure spaces) which preserves their measures is generated by a point transformation preserving measure. This result is important in showing the equivalence of rather general measure spaces, when they are separable and complete, to Euclidean spaces with Lebesgue measure, and permits one to reduce the study of Boolean algebras of measurable sets to ordinary measures.

In [51] von Neumann proves the uniqueness of the Haar measure as constructed by A. Haar (in Ann. of Math. vol. 34, pp. 147-169), if one requires either left or right invariance of the (Lebesgue-type) measure under group multiplication. The theorem on uniqueness is proved for compact groups. A construction different from that of Haar is employed to introduce his measure. This paper precedes the construction of a general theory of almost periodic functions on separable topological groups and allows a theory of their orthogonal representations.

- Recently pushed to the most extreme minimal form by R. M. Robinson. 
In paper [54] the ordinary notion of completeness, usually defined only for metric spaces, is generalized for linear topological spaces. Interesting examples of spaces which are not metric but complete are produced. Such cases involve, of course, non-separable spaces. The paper also contains a novel construction of pseudo-metrics and convex spaces.

In a joint paper with P. Jordan [59], a solution is given to a question raised by Fréchet of the characterization of generalized Hilbert space among linear metric spaces. The condition which is necessary and sufficient, strengthening a result of Fréchet, is: A linear metric space $L$ is isometric with a Hilbert space if and only if every 2-dimensional linear subspace is isometric with a Euclidean space.

The results of [35] are generalized in a joint paper with $M . H$. Stone [60] and deal with selection of representative elements from residual classes in an abstract ring modulo a given left- and rightideal. The article contains a number of theorems on representations of Boolean rings modulo an ideal.

In the Russian "Sbornik," [64], von Neumann deals again with the problem of the uniqueness of Haar's measure. The previous proof of uniqueness was accomplished through a constructive process different from that of Haar, which contained no arbitrary elements and led automatically to the uniqueness of the measure. In this paper an independent treatment of uniqueness of the left- and right-invariant exterior measure is given for locally compact separable groups. (A different proof was obtained simultaneously by André Weil.)

In a joint paper with Kuratowski, [69], precise and strong results are obtained on the projectivity of certain sets of real numbers defined by transfinite induction. The celebrated set of Lebesgue, ${ }^{7}$ shown previously by Kuratowski to be of projective class 3, is shown to be a difference of two analytic sets and therefore of the second projective class. A general theorem is proved on the analytic character of sets (in the sense of Hausdorff) obtained by certain general constructions. This result is likely to play an important role in the still incomplete theory of projective sets.

The Memoire in Compositio Mathematica, [75], on infinite direct products contains an algebraic theory of operators and a measure theory for such systems, so important in modern abstract analysis. It summarizes some of the previous work on the algebra of functional operators and topology of rings of operators, including the nonseparable hyper-Hilbert spaces. Methodologically and in the actual constructions, this paper is both a forerunner of and a good introduc-

${ }^{7}$ Journal de Mathématiques, 1905, Chapter VIII. 
tion to much of the recent work in mathematics dealing, so to say, with the pyramiding of algebraical notions. Starting with a vector space, one deals first with their products, then with linear operators on these structures; and finally with classes of such operators whose algebraical properties are investigated again "on the first level." Von Neumann intended to discuss the analogy of this elaborate system with the theory of hyperquantization in quantum theory, and considered the paper in particular as a mathematical preparation for dealing with non-enumerable products.

The paper [24] is, I believe, the first one in which a very significant contribution is made to the complex of questions originating in Hilbert's fifth problem: the possibility of a change of parameters in a continuous group so that the group operation will become analytic. The work deals with subgroups of the group of linear transformations of $n$-dimensional space and the result is affirmative: Every such continuous group has a normal subgroup, locally representable analytically and in a one-to-one way by a finite number of parameters.

This is the first of the theorems showing that the group property prevents the "pathological" possibilities common in the theory of functions of a real variable. The results of the paper, later generalized and simplified by E. Cartan for subgroups of general Lie groups, give detailed insight into the structure of such groups by the representation of elements as products of exponential operators. They show that every linear manifold which contains with every two matrices $U, V$ also their commutator $U V-V U$, is an infinitesimal group of an entire group $G$. This paper is historically important, as preceding the work of Cartan, a later paper of Ado, and of course, von Neumann's own paper [48] where Hilbert's fifth problem is solved for compact groups.

This celebrated result is based on and stimulated by a paper of Haar (in the same volume of Annals of Math.) where an invariant measure function is introduced in continuous groups. Von Neumann shows, (using an analogue of the Peter-Weyl integration on groups and employing the theorem on approximability of functions by linear combinations of a finite number of eigenfunctions of an integral operator-the method of E. Schmidt's dissertation-and with an ingenious use of Brouwer's theorem on invariance of region in Euclidean $n$-dimensional space) - that every compact and $n$-dimensional topological group is continuously isomorphic to a closed group of unitary matrices of a finite dimensional Euclidean space.

The method of this article allows one to represent more general (not necessarily $n$-dimensional) groups as subgroups of infinite products of such $n$-dimensional groups. In the second part of the 
paper, an example is given of a finite dimensional non-compact group of transformations acting on Euclidean space in such a way that no change of parameters in the space will make the given transformations analytic. It was almost twenty years before the solution of Hilbert's fifth problem was completed, to include the "open" (i.e., non-compact) $n$-dimensional groups, by the work of Montgomery and Gleason. Von Neumann's achievement required an intimate knowledge of both the set-theoretical, real variable techniques, a feeling for the spirit of Brouwerian topology, and a real understanding of the technique of integral equations and the calculus of matrices.

A combination of virtuosity in the mode of abstract algebraic thinking and the employment of analytical techniques can be seen in the joint paper [50] with Jordan and Wigner on an algebraic generalization of the quantum mechanical formalism. This is conceived as a possible starting point for future generalizations of the quantum mechanical theories and deals with commutative but not associative hypercomplex algebras. The essential result is that all such formally real finite and commutative $r$-number systems are merely matrix algebras, with one exception. This exception, however, seems too narrow for the generalizations needed in quantum theories.

An unpublished result, announced in the Bulletin of the American Mathematical Society [14, Appendix 2] contains the theorem on the simplicity (of the component of unity) of the group of all homeomorphisms of the surface of the 3-dimensional sphere. The actual theorem is that, given two arbitrary homeomorphisms $A, B$ (neither equal to identity) - there exists a fixed number (23 is sufficient!) of conjugates of the first one whose product is equal to $B$.

Hilbert space, operator, theory, rings of operators. A detailed account of von Neumann's fundamental and comprehensive treatment of these topics is presented in the articles in this volume by Professor Murray and Professor Kadison. His first interest in this subject also stemmed from work on rigorous formulations of quantum theory. In 1954, in a questionnaire which von Neumann answered for the National Academy of Sciences, he named this work as one of his three contributions to mathematics that he considered most important. In sheer bulk alone, papers on these subjects comprise roughly one-third of his printed work. These contain a very detailed analysis of properties of linear operators and an algebraical study of classes (rings) of operators in infinite-dimensional spaces. The result fulfills his avowed purpose stated in the book, Mathematische Grundlagen der Quantenmechanik [47a], of demonstrating that the ideas originally introduced by Hilbert are capable of constituting 
an adequate basis for the physical considerations of quantum theory, and that no need exists for the introduction of new mathematical schemes for these physical theories. Von Neumann's unbelievably detailed and meticulous work of classification of the properties of linearity for unitary spaces resolves many problems for unbounded operators. It gives a complete theory of hypermaximal transformations and brings Hilbert space almost as completely within the grasp of the mathematician as is the case with the finite dimensional Euclidean space.

His interest in this subject was continuous throughout his scientific life. Even up to the end, in the midst of work on other subjects, he obtained and published results on the properties of operators and spectral theory. Paper [106] was published in 1950, and written in honor of the 75th birthday of Erhard Schmidt. (It was Schmidt who first introduced him to the fascinations of this subject.) No one has done more than von Neumann, at least in the unitary case and for linear transformations, towards the resolution of the mysteries of non-compactness. Future work in this direction will be based on his results for a long time to come. This work is now being vigorously continued by, among others, his collaborators and former studentsMurray in particular - and one is entitled to expect from them further valuable insight into the properties of linear operators.

Theory of lattices, continuous geometry. Birkhoff's article, von Neumann and lattice theory, presents the work on these subjects. Here again, von Neumann's interest was stimulated by the possibility of applying these new combinatorial and algebraic schemes to quantum theory. Lattice theory, around 1935, was being developed and generalized by Garrett Birkhoff from the original formulations of Dedekind. At about the same time, an algebraic and set-theoretical study of Boolean algebras was systematically undertaken by M. H. Stone. I remember that in the summer of 1935, Birkhoff, Stone, and von Neumann, on their way from a mathematical meeting in Moscow, stopped in Warsaw and presented short talks at a meeting of the Warsaw Mathematical Society on the new developments in these fields with novel formulations of the logic of quantum theory. The ensuing discussions led one to expect far-reaching applications of the general Boolean Algebra and lattice theory formulations of the language of quantum theory. Von Neumann returned to these attempts several times later in his work, but most of his thoughts in this direction are in unpublished notes. ${ }^{8}$

${ }^{8}$ Professor Givens is preparing an edition of the lecture notes to be published shortly by the Princeton Press. Another paper on continuous geometry written in 1935 is being published in the Annals of Mathematics. 
His work on continuous geometries and geometries without points was motivated by the belief that the primitive notions of quantum theory deal with such entities; obviously, the "universe of discourse" consists of certain classes of identified points or linear manifolds in Hilbert space. (This is noted explicitly by Dirac in his book.)

Some of this work was considered for presentation in colloquium lectures; an account of it is contained in the Princeton Institute Lectures; some remains in manuscript form. In conversations with him touching upon these problems, my impression was that, beginning about 1938, von Neumann felt that the new facts and problems of nuclear physics gave rise to problems of an entirely different type and made it less important to insist on a mathematically flawless formulation of a quantum theory of atomic phenomena alone. After the end of the war, he would express sentiments, somewhat similar to remarks reportedly made by Einstein, that the bewildering wealth of nuclear and elementary particle physics make premature any attempt to formulate a general quantum theory of fields, at least for the time being.

Theoretical physics. Professor Van Hove describes von Neumann's work in Von Neumann's contributions to quantum theory.

In the questionnaire for the National Academy of Science mentioned earlier, von Neumann selected as his most important scientific contributions work on mathematical foundations of Quantum Theory and the Ergodic Theorem (in addition to the Theory of Operators discussed above). This choice, or rather restriction, might appear curious to most mathematicians, but is psychologically interesting. It seems to indicate that perhaps his main desire and one of his strongest motivations was to help re-establish the role of mathematics on a conceptual level in theoretical physics. The drifting apart of abstract mathematical research and of the main stream of ideas in theoretical physics since the end of the First World War is undeniable. Von Neumann of ten expressed concern that mathematics might not keep abreast of the exponential increase of problems and ideas in physical sciences. I remember a conversation in which I advanced the fear that a sort of Malthusian divergence may take place: the physical sciences and technology increase in a geometrical ratio and mathematics in an arithmetical progression. He said that this indeed might be the case. Later in the discussion, we both managed to cling, however, to the hope that the mathematical method would remain for a long time in conceptual control of the exact sciences!

Article [7] is published jointly with Hilbert and Nordheim. According to the preface, it is based on a lecture given by Hilbert in the 
winter of 1926 on the new developments in quantum theory, and prepared with the help of Nordheim. According to the introduction, important parts of the mathematical formulation and discussion are due to von Neumann.

The stated aim of the paper is to introduce, instead of strictly functional relationships of classical mechanics, probability relationships. It also formulates the ideas of Jordan and Dirac in a considerably simpler and more comprehensible manner. Even now, 30 years later, it is difficult to overestimate the historical importance and influence of this paper and the subsequent work of von Neumann in this direction. The great program of Hilbert in axiomatization gains here another vital domain of application, an isomorphism between a physical theory and the corresponding mathematical system. An explicit statement in the introduction to the paper is that it is difficult to understand the theory if its formalism and its physical interpretation are not separated concisely and completely. Such separation is the aim of the paper, even though it is admitted that a complete axiomatization was at the time impossible. May we add here parenthetically that such complete axiomatization of a relativistically invariant quantum theory, embracing its application to nuclear phenomena is still to be achieved. ${ }^{9}$ The paper contains an outline of the calculus of operators which correspond to physical observables, discusses the properties of Hermitean operators, and altogether forms a prelude to the Mathematische Begrundung der Quantenmechanik.

Von Neumann's precise and definitive ideas on the role of statistical mechanics in quantum theory and the problem of measurement are introduced in [10].

His well-known book, [47a], gives both the axiomatic treatment, the theory of measurement, and statistics in detailed discussions.

At least two mathematical contributions are of importance in the history of quantum mechanics: The mathematical treatment by Dirac did not always satisfy the requirements of mathematical rigor. For example, it operated with the assumption that every self-adjoint operator can be brought into diagonal form, which forced one to introduce for those operators where this cannot be done, the famous "improper" functions of Dirac. A priori it might seem, as von Neumann states, that just as Newtonian mechanics required (at that

\footnotetext{
${ }^{9}$ For an excellent succinct summary of the present state of axiomatizations of non-relativistic quantum theory in the domain of atomic phenomena, see the article by George Mackey, Quantum mechanics and Hilbert space, Amer. Math. Monthly, October, 1957, still based essentially on von Neumann's book, Mathematische Grundlagen der Quantenmechanik.
} 
time) the contradictory infinitesimal calculus, so quantum theory seemed to need a new form of analysis of infinitely many variables. Von Neumann's achievement was to show that this was not the case, namely, that the transformation theory could be put on a clear mathematical basis not by making precise the methods of Dirac but by developing Hilbert's spectral theory of operators. In particular, this was accomplished by his study of non-bounded operators going beyond the classical theory of Hilbert, F. Riesz, E. Schmidt, and others.

The second contribution forms the substance of Chapters 5 and 6 of his book. It has to do with the problems of measure and reversibility in quantum theory. Almost from the beginning, when the ideas of Heisenberg, Schrödinger, Dirac, and Born were enjoying their first sensational success, questions were raised on the role of indeterminism in the theory and proposals made to explain it by the assumption of possible "hidden" parameters which, when discovered in the future, would allow a return to a more deterministic description. Von Neumann shows that the statistical character of statements of the theory is not due to the fact that the state of the observer who performs the measurement is unknown. The system comprising both the observed and observer leads to the uncertainty relations even if one admits an exact state of the observer. This is shown to be the consequence of the previous assumptions of quantum theory involving the general properties of association of physical quantities with operators in Hilbert space. ${ }^{10}$

Apart from the great didactic value of this work which presented the ideas of the new quantum theory in a form congenial and technically interesting to mathematicians, it is a contribution of absolutely first importance, considered as an attempt to make a rational presentation of a physical theory which, as originally conceived by the physicists, was based on non-universally communicable intuitions. While it cannot be asserted that it introduced ideas of novel physical import-and the quantum theory as conceived during these years by Schrödinger, Heisenberg, Dirac, and others still forms only an incomplete theoretical skeleton for the more baffling physical phenomena discovered since-von Neumann's treatment allows at

${ }^{10} \mathrm{It}$ is impossible to summarize here the mathematical argument involved. The great majority of physicists still agree with von Neumann's proposition. This is not to say that a theory different from the present mathematical formulations of quantum mechanics might not allow such a role for hidden parameters. For a recent discussion, see Volume 9 of the Colston Papers, being the Proceedings of the Ninth Symposium of the Colston Research Society held in the University of Bristol, April 1-April 4, 1957, discussions of Bohm, Rosenfeld, et al. 
least one logically and mathematically clear basis for a rigorous treatment.

Analysis, numerical work, work in hydrodynamics. An early paper is [33]. In it, a fundamental lemma in the calculus of variations due to Radó is proved by means of a simple geometrical construction (the lemma asserts that a function $z=f(x, y)$ satisfies a Lipschitz condition with a constant $\Delta$ if no plane whose maximal inclination is greater than $\Delta$ meets the boundary of the surface defined by the given function in three or more points). The paper is also interesting in that the method of proof involves direct geometric visualizations somewhat rare in von Neumann's published work.

The paper [41] contains one of the impressive achievements of mathematical analysis in the last quarter century. It is the first precise mathematical result in a whole field of investigation: a rigorous treatment of the ergodic hypothesis in statistical mechanics. It was stimulated by the discovery by Koopman of the possibility of reducing the study of Hamiltonian dynamical systems to that of operators in Hilbert space. Using Koopman's representation, von Neumann proved what is now known as the weak ergodic theorem, or the convergence in measure of the means of functions of the iterated, measure-preserving transformation on a measure space. It is this theorem, strengthened shortly afterwards by G. D. Birkhoff, in the form of convergence almost everywhere, which provided the first rigorous mathematical basis for the foundations of classical statistical mechanics. The subsequent developments in this field and the numerous generalizations of these results are well-known and will not be mentioned here in detail. Again, this success was due to the combination of von Neumann's mastery of the techniques of the set-theoretically inspired methods of analysis and those originating in his own work on operators on Hilbert space. Still another domain of mathematical physics became accessible to precise and general considerations of modern analysis. In this instance again, a great initial advance was scored, but, of course, here the story is really quite unfinished; a mathematical treatment of the foundations of statistical mechanics, in the case of classical dynamics, is far from complete! It is very well to have the ergodic theorems and the knowledge of the existence of metrically transitive transformations; these facts, however, form only a basis of the subject. Von Neumann often expressed in conversations a feeling that future progress will depend on theorems which would allow a mathematically satisfactory treatment of the subsequent parts of the subject. A complete mathematical theory of the 
Boltzmann equation and precise theorems on the rates at which systems tend towards equilibrium are needed.

Important work is contained in the article [56], a joint work with S. Bochner. The use of operator-theoretical methods allows a rather profound discussion of the properties of partial differential equations of the type $A \phi=\partial \phi / \partial t, \phi=\phi(t ; x, y, z)$, with $A$ of the form

$$
A=a\left(\frac{\partial^{2}}{\partial x^{2}}+\frac{\partial^{2}}{\partial y^{2}}+\frac{\partial^{2}}{\partial z^{2}}\right)
$$

as in problems of heat conduction, or $A=(2 \pi i / h) H$, where $H$ is the energy operator in Schrödinger's quantum mechanical equation for non-stationary states.

An example of the combination of analytical and geometrical techniques is the joint work with Schoenberg [80]. If $S$ is a metric space, $d(f, g)$ being the distance between any two elements of it, we call a function, $f_{t}$, whose values lie in $S$ and which is continuous, a screw function if $d\left(f_{t}, f_{s}\right)=F(t-s)$. The fundamental theorem determines the class of all such functions on a Hilbert space and determines their form. (Any such function $F(t)$ is given by

$$
F^{2}(t)=\int_{0}^{\infty} \frac{\sin ^{2} t u}{u^{2}} d \gamma(u)
$$

where $\gamma(u)$ is non-decreasing for $u \geqq 0$ and such that $\int_{1}^{\infty} u^{-2} d \gamma(u)$ exists.)

The paper [86], perhaps less well-known than it deserves to be, shows an increasing interest in approximation problems and in numerical work. It seems to me of very considerable didactical value. It deals with properties of finite $N \times N$ matrices for large $N$. The behavior of the space of all linear operations on the $N$-dimensional complex Euclidean space is investigated. This is done in detail directly, and it is stated explicitly in the preface that such an asymptotic approach has been unjustifiably neglected compared to the usual approach which is the study of the limiting case, i.e., the actually infinitely dimensional unitary space, that is to say, Hilbert space. (It is curious to contrast this statement with the almost opposite point of view expressed in the introduction to his book, Mathematische Grundlagen der Quantenmechanik.)

In general terms, the paper deals with the question of which $N$ th order matrices behave or behave approximately as if they were $m$ th order matrices. ( $m$ being small compared to $N$ and a divisor of it.) The notion of approximate behavior is made precise in a given metric or pseudo metric in the space of matrices. I should like to add that 
this paper has a praiseworthy elementary character of exposition not always found in his work on Hilbert space.

Work belonging to this same order of ideas is continued in his joint paper [91] with Bargmann and Montgomery. It contains an account of various methods of solving a system of linear equations and is oriented towards the possibilities, already beginning to appear at that time, of computations involving the use of electronic machines.

In problems of applied analysis, the war years brought a need for quick estimates and approximate results in problems which often do not present a very "clean" appearance, that is to say, are mathematically very inhomogeneous, the physical phenomena to be calculated involving, in addition to the main process, a number of external perturbations whose effect cannot be neglected or even separated in additional variables. This situation comes up often in questions of present day technology and forces one, at least initially, to resort to numerical methods, not because one requires the results with high accuracy but simply to achieve qualitative orientation! This fact, perhaps somewhat deplorable for a mathematical purist, was realized by von Neumann whose interest in numerical analysis increased greatly at that time.

A joint work with H. H. Goldstine, [94], presents a study of the problem of the numerical inversion of matrices of high order. Among other things, it attempts to give rigorous error estimates. Interesting results are obtained on the precision achievable in inverting matrices of order 150. Estimates are obtained "in the general case." ("General" means that under plausible assumed statistics, these estimates hold with the exception of a set of low probability.)

In a subsequent paper on this subject, [109], the problem is reconsidered in an effort to obtain optimum numerical estimates. Given a matrix $A=\left(a_{i j}\right)(i, j=1,2, \cdots n)$ whose elements are independent random variables, each normally distributed, the probability that the upper bound of this matrix exceeds $2.72 \sigma n^{1 / 2}$ where $\sigma$ is the dispersion of each variable, is less than $.027 \times 2^{-n} n^{-1 / 2}$.

The development of the fast electronic computing machines was prompted primarily by the need of a quick orientation and answer to problems in mathematical physics and engineering. There is, as a byproduct, an opportunity for some lighter work! Thus, for example, one can now try to satisfy, to a modest extent, some of the curiosity which is felt about certain interesting sequences of integers, e.g., to mention the simplest ones, the frequency of the sequence of digits in the development of $e$ and $\pi$, carried to many thousands of places. 
One such computation, performed on the machine at the Institute for Advanced Study, gives the first 2,000 partial quotients of the cube root of 2 in its development as a continued fraction. Johnny was interested in such experimental work no matter how simple-minded the problem; in one discussion in Los Alamos on such questions, he asked to be given "interesting" numbers for computation of their continued fraction development. I named the quartic irrationality $y$ given by the equations $y=1 /(x+y)$ where $x=1 /(1+x)$ as one in whose development there might appear some curious regularities. Computations of many other numbers were planned, but it is not known to me whether this little project was ever pursued.

Game theory. This subject forms a new, rapidly developing chapter in present-day mathematical research; it is essentially a creation of von Neumann's. His fundamental work in this field will be described elsewhere in this volume by A. W. Tucker and H. W. Kuhn and I shall content myself with remarking that it presents some of his most fecund and influential work. It was Borel, in a note in the Comptes-Rendus in 1921, who first formulated a mathematical scheme describing strategies in a game between two players. The subject can, however, be dated as really originating in the paper of von Neumann, [17]. It is there that the fundamental "minimax" theorem is proved and the general scheme of a game between $n$ players $(n \geqq 2)$ is formulated. Such schemata, quite apart from their interest and applications to actual games in economics, etc. introduced a wealth of novel combinatorial problems of purely mathematical interest. The theorem that Min Max $=$ Max Min and the corollaries on the existence of saddle points of functions of many variables is contained in his 1937 paper [72]. They are shown to be a consequence of a generalization of Brouwer's fixed-point theorem and of the following geometrical fact. Let $S, T$ be two non-empty, convex, closed, and bounded sets contained in the Euclidean spaces $R_{n}$ and $R_{m}$ respectively. Let $S \times T$ be the direct product of these sets and $V$, $W$ two closed subsets of it. Assume that for every element $x$ of $S$ the set $Q(x)$ of all $y$ such that $(x, y)$ belongs to $V$ is a closed convex and non-empty set. Analogously, for every $y$ in $T$ the set $P(y)$ of all $x$ such that $(x, y)$ belongs to $W$ also has this property. Then the sets $V$ and $W$ have at least one point in common. This theorem, further discussed by Kakutani, Nash, Brown and others, plays a central role in the proofs of existence of "good strategies."

Game theory, including now a study of infinite games (first formulated by Mazur in Poland around 1930) is in vigorous mathematical development. It suffices to refer to the work contained in the three volumes, Contributions to Game Theory $[102 ; 113 ; 114]$, to point 
out the wealth of ideas, the variety of ingenious formulations in purely mathematical context, and the increasing number of important applications; it abounds in simply stated problems still unsolved.

Economics. The now classical treatise by Oskar Morgenstern and John von Neumann, Theory of games and economic behavior [90] contains an exposition of Game Theory in its purely mathematical form with a very detailed account of applications to actual games; and together with a discussion of some fundamental questions of economic theory introduces a different treatment of problems of economic behavior and certain aspects of sociology. The economist Oskar Morgenstern, a friend of von Neumann's in Princeton for many years, interested him in aspects of economic situations, specifically in problems of exchange of goods between two or more persons, in problems of monopoly, oligopoly and free competition. It was in a discussion of attempts to schematize mathematically such processes that the present shape of this theory began to take form.

The present numerous applications to "operational research," problems of communications and the statistical estimation theory of A. Wald either stem from or are drawing upon the ideas proposed and worked out in this monograph. We cannot outline in this article even the scope of these investigations. The interested reader may find an account of it in, e.g., L. Hurwicz's The theory of economic behavior ${ }^{11}$ and J. Marshak's Neumann's and Morgenstern's new approach to static economics. ${ }^{12}$

Dynamics, mechanics of continua, meteorological calculations. In two papers written jointly with S. Chandrasekhar [84 and 88] the following problem is considered. A random distribution of mass centers is assumed; these might be, for example, stars in a cluster or a cluster of nebulae. These masses are mutually attracting and in motion. The problem is to develop the statistics of the fluctuating gravitational field and the study of the motions of individual masses subject to the changing influence of the varying local distributions. In the first paper, the problem of the rate of the fluctuations in the distribution function for the force is solved through ingenious calculations, and a general formula is obtained for the probability distributions $W(F, f)$ of a gravitational field strength $F$ and an associated rate of change $f$ which is the derivative of $F$ with respect to time. Among the results obtained is the theorem that for weak fields the

11 American Economic Review vol. 35 (1945) pp. 909-925.

12 Journal of Political Economy vol. 54 (1946) pp. 97-115. 
probability of a change occurring in the field acting at a given instant of time is independent of the direction and magnitude of the initial field, while for strong fields, the probability of a change occurring in the direction of the initial field is twice as great as in a direction at right angles to it.

The second paper is devoted to a statistical analysis of the speed of fluctuations in the force per unit mass acting on a star which moves with a velocity $V$ with respect to the centroid of the nearby stars. This problem is solved on the assumption of a uniform Poisson distribution of the stars and a spherical distribution of the local velocities. It is solved for a general distribution of different masses. An expression is derived for the correlations in the force acting at two very close points. The method gives the asymptotic behavior of the space correlations. Von Neumann was long interested in the phenomenon of turbulence. The writer remembers discussions in 1937 on the possibility of a statistical treatment of the Navier-Stokes equations by an analysis of hydrodynamical problems through replacement of the partial differential equations by a system of infinitely many total differential equations satisfied by the Fourier coefficients in the development of the Lagrangian functions in a Fourier series. A mimeographed report written by von Neumann for the Office of Naval Research in 1949, Recent theories of turbulence, constitutes a penetrating and lucid presentation of the ideas of Onsager and Kolmogoroff, and of other work up to that time.

With the beginning of the second World War, von Neumann undertook a study of problems presented by the motions of compressible gases and especially the perplexing phenomena of formation of discontinuities, e.g., shocks.

The greater part of his voluminous study in this field was prompted by problems arising in defense work. They were published in the form of reports. A selection is included in the bibliography.

It is impossible to summarize here this varied work; most of it is characterized by his incisive analytical technique and the accustomed clarity of logic. In the theory of interaction of colliding shocks, his contributions are especially noteworthy. One result is the first rigorous justification of the Chapman-Jouguet hypothesis concerning the process of detonation, that is, a combustion process initiated by a shock.

The first systematic development of the theory of reflection of shock waves was initiated by von Neumann (Progress report on the theory of shock wave, NDRC, Div. 8, OSRD, No. 1140, 1943 and Oblique reflection of shocks, Navy Department, Explosive Research Report no. 12, 1943). 
As noted before, the problem of following, even only qualitatively, the motions of compressible media in two or three dimensions surpasses the present powers of explicit analysis. What is worse, the mathematical foundations of a theory which would describe the physical phenomena are, perhaps so far, quite inadequate. Von Neumann's feelings in this matter are well expressed in comments contained in [108]:

"The question as to whether a solution which one has found by mathematical reason really occurs in nature and whether the existence of several solutions with certain good or bad features can be excluded beforehand, is a quite difficult and ambiguous one. This subject has been considered in the classical literature as well as in the more recent literature, on widely varying levels of rigor and of its opposite. In summa, it is quite difficult ever to be sure of anything in this domain. Mathematically, one is in a continuous state of uncertainty, because the usual theorems of existence and uniqueness of a solution, that one would like to have, have never been demonstrated and are probably not true in their obvious forms."

and later,

"Thus there exists a wide variety of mathematical possibilities in fluid mechanics, with respect to permitting discontinuities, demanding a reasonable thermodynamic behavior etc., etc. There probably exists a set of conditions under which one and only one solution exists in every reasonably stated problem. However, we have only surmises as to what it is and we have to be guided almost entirely by physical intuition in searching for it. It is therefore impossible to be very specific about any point. And it is difficult to say about any solution which has been derived, with any degree of assurance, that it is the one which must exist in nature."

One has to resort to numerical work in special cases if only to get a heuristic insight into these difficult questions. In a whole series of reports, von Neumann discussed the best numerical procedures, differencing schemes, questions of numerical stability of computational schemes for such calculations. One should mention in particular the paper [100] with Richtmyer, where, in order not to introduce explicitly the shock conditions and discontinuities, a purely mathematical, fictitious viscosity is introduced, allowing one to proceed to calculate the motion of shocks without postulating them explicitly but following step by step the ordinary hydrodynamic equations.

The formidable mathematical problems presented by the hydrodynamical equations of the motions of the earth's atmosphere fascinated von Neumann for a considerable time. With the advent of computing machines, a detailed numerical study at least of simplified versions of the problems became possible, and a large program of such work was started by him. At the Institute in Princeton, a meteorological research group was established $;^{13}$ the plan was to

${ }^{18} \mathrm{~J}$. Charney was working closely with him on problems of meteorology, e.g., paper [104]. 
attack the problem of numerical weather solution by a step-by-step investigation of models which were to approximate more and more closely the real properties of the atmosphere. A numerical investigation of truly 3-dimensional motions is at present impractical even on the most advanced electronic computing machines. (This may not be the case, say five years from now.)

The first highly schematized computations which von Neumann initiated dealt with a 2-dimensional model and for the most part in the so-called geostrophic approximation. Later, what might be called " $2+1 / 2$ " dimensional hydrodynamical computations were performed by assuming two or three 2-dimensional models corresponding to different altitudes or pressure levels interacting with each other. This problem was dear to his mind, both because of its intrinsic mathematical interest, and because of the enormous technological consequences which a successful solution could have. He believed that our knowledge of dynamics of controlling processes in the atmosphere, together with the development of computing machines, was approaching a level that would permit weather prediction. Beyond that, he believed that one could understand, calculate, and perhaps put into effect processes ultimately permitting control and change of the climate.

In the paper [120] he speculated on the approach of the time when one could produce, with the now available vast nuclear sources of energy, changes in the general circulation of the atmosphere of the same order of magnitude as "the great globe itself." In such problems where the physics of the phenomena are already understood, it might be that a future Mathematical Analysis will enable the human race to extend vastly its control over nature.

Theory and practice of computing on electronic machines, Monte Carlo method. Von Neumann's interest in numerical work had different sources. One stemmed from his original work on the role of formalism in mathematical logic and set-theory, and his youthful work was concerned extensively with Hilbert's program of considering mathematics as a finite game. Another equally strong motivation came from his work in problems of mathematical physics including the purely theoretical work on ergodic theory in classical physics and his contributions to quantum theory. A growing exposure to the more practical problems encountered in hydrodynamics and in the manifold problems of mechanics of continua arising in the technology of nuclear energy led directly to problems of computation.

We have already briefly discussed his interest in the problems of 
turbulence, general dynamics of continua, and meteorological calculations.

I remember quite well how, very early in the Los Alamos Project, it became obvious that analytical work alone was of ten not sufficient to provide even qualitative answers. The numerical work by hand and even the use of desk computing machines would require a prohibitively long time for these problems. This situation seemed to provide the final spur for von Neumann to engage himself energetically in the work on methods of computation utilizing the electronic machines.

For several years von Neumann had felt that in many problems of hydrodynamics-in propagation and the behavior of shocks, and generally in cases where the non-linear partial differential equations describing the phenomena had to be applied in instances involving large displacements (that is to say, in cases where linearization would not adequately approximate the true description) numerical work was necessary to provide heuristic material for a future theory.

This final necessity compelled him to examine, from its foundations, the problem of computing on electronic machines and, during 1944 and 1945, he formulated the now fundamental methods of translating a set of mathematical procedures into a language of instructions for a computing machine. The electronic machines of that time (e.g., the Eniac) lacked the flexibility and generality which they now possess in the handling of mathematical problems. Speaking broadly, each problem required a special and different system of wiring, in order to enable the machine to perform the prescribed operations in a given sequence. Von Neumann's great contribution was the idea of a fixed and rather universal set of connections or circuits in the machine, a "flow diagram," and a "code" so as to enable a fixed set of connections in the machine to have the means of solving a very great variety of problems. While, a priori at least, the possibility of such an arrangement might be obvious to mathematical logicians, the execution and practice of such a universal method was far from obvious with the then existing electronic technology.

It is easy to underestimate, even now, ten years after the inception of such methods, the great possibilities opened through such theoretical experimentation in problems of mathematical physics. The field is still new and it seems risky to make prophesies, but the already accumulated mass of theoretical experiments in hydrodynamics, magneto-hydrodynamics, and quantum-theoretical calculations, etc., allow one to hope that good syntheses may arise from these computations. 
The engineering of the computing machines owes a great deal to von Neumann. The logical schemata of the machines, the planning of the relative roles of their memory, their speed, the selection of fundamental "orders" and their circuits in the present machines bear heavily the imprint of his ideas. Von Neumann himself supervised the construction of a machine at the Institute for Advanced Study in Princeton, so as to have an acquaintance with the engineering problems involved and at the same time to have at hand this tool for novel experimentation. Even before the machine was finished, which took longer than anticipated, he was involved in setting up and executing enormous computations arising in certain problems at the Los Alamos Laboratory. One of these, the problem of following the course of a thermonuclear reaction, involved more than a billion of elementary arithmetical operations and elementary logical orders. The problem was to find a "yes" or "no" answer to the question of propagation of a reaction. One was not concerned with providing the final data with great accuracy but, in order to obtain an answer to the original question, all the intermediate and detailed computations seemed necessary. It is true that guessing the behavior of certain elements of the problem, together with hand calculations, could indeed throw considerable light on the final answer. In order to increase the degree of confidence in estimates thus obtained by intuition, an enormous amount of computational work had to be undertaken. This seems to be rather common in some new problems of mathematical physics and of modern technology. Astronomical accuracy is not required in the description of the phenomena; in some cases, one would be satisfied with predicting the behavior "up to 10 percent" and yet during the course of the calculations, the individual steps have to be kept as accurate as possible. The enormous number of elementary steps then poses the problem of estimating the reliability of final results and problems on the intrinsic stability of mathematical methods and their computational execution.

In receiving the Fermi prize of the Atomic Energy Commission, von Neumann was cited especially for his contribution to the development of computing on the electronic machines, so useful in many aspects of nuclear science and technology.

The electronic computing machines with their speed of computation surpassing that of the hand calculations by a factor of many thousands invite the invention of entirely new methods not only in numerical analysis in the classical sense, but in the very foundations of procedures of mathematical analysis itself. Nobody was more 
aware of these implications than von Neumann. A small example of what we mean here can be illustrated by the so-called Monte Carlo Method. The methods of numerical analysis as developed in the past for hand work, or even for the relay machines, are not necessarily optimal for computations on the electronic machines. So, for example, it is obvious that instead of employing tables of elementary functions, it is more economical to compute the desired values directly. Next, it is clear that the procedures of integration of equations by reduction to quadratures, etc., can now be circumvented by schemes so complicated arithmetically that they could not even be considered for hand work, but which are very feasible on the new machines. Literally dozens of computational tricks, "subroutines," e.g., for calculating elementary algebraical or transcendental functions, for solving of auxiliary equations, etc. were produced by von Neumann during the years following the World War. Some of this work, by the way, is not as yet generally available to the mathematical public, but is more widely known among the now numerous technological and scientific groups utilizing the computing machines in industrial or government projects. This work includes methods for finding eigenvalues and inversion of matrices, methods for economical search for extrema of functions of several variables, production of random digits, etc. Much of this exhibits the typical combinatorial dexterity, in some cases, of virtuoso quality, of his early work in mathematical logic and algebraical studies in operator theory.

The simplicity of mathematical formulation of the principles of mathematical physics hoped for in the nineteenth century seems to be conspicuously absent in modern theories. A perplexing variety and wealth of structure found in what one considered as elementary particles, seem to postpone the hopes for an early mathematical synthesis. In applied physics and in technology one is forced to deal with situations which, mathematically, present mixtures of different systems: For example, in addition to a system of particles whose behavior is governed by equations of mechanics, there are interacting electrical fields, described by partial differential equations; or, in the study of behavior of neutron-producing assemblies, one has, in addition to a system of neutrons, the hydrodynamical and the thermodynamical properties of the whole system interacting with the discrete assembly of these particles.

From the point of view of combinatorics alone, not to mention the difficulties of analysis in the handling of several partial differential and integral equations, it is clear that at the present time, there is 
very little hope of finding solutions in a closed form. In order to find, even only qualitatively, the properties of such systems, one is forced to look for pragmatic methods.

We decided to look for ways to find, as it were, homomorphic images of the given physical problem in a mathematical schema which could be represented by a system of fictitious "particles" treated by an electronic computer. It is especially in problems involving functions of a considerable number of independent variables that such procedures would be applied. To give a very simple concrete example of such a Monte Carlo approach, let us consider the question of evaluating the volume of a subregion of a given $n$-dimensional "cube" described by a set of inequalities. Instead of the usual method of approximating the volume required by a systematic subdivision of the space into its lattice points one could select, at random, with uniform probability, a number of points in space and determine (on the machine) how many of these points belong to the given region. This proportion will give us, according to elementary facts of probability theory, an approximate value of the relative volumes, with the probability as close to one as we wish, by employing a sufficient number of sample points. As a somewhat more complicated example, consider the problem of diffusion in a region of space bounded by surfaces which partly reflect and partly absorb the diffusing particles. If the geometry of the region is complicated, it might be more economical to try to perform "physically" a large number of such random walks rather than to try to solve the integro-differential equations classically. These "walks" can be performed conveniently on machines and such a procedure in fact reverses the treatment which in probability theory reduces the study of random walks to the study of differential equations.

Another instance of such methodology is, given a set of functional equations, to attempt to transform it into an equivalent one which would admit of a probabilistic or game theory interpretation. This latter would allow one to play, on a machine, the games illustrating the random processes and the distributions obtained would give a fair idea of the solution of the original equations. Better still, the hope would be to obtain directly a "homomorphic image" of the behavior of the physical system in question. It has to be.stated that in many physical problems presently considered, the differential equations originally obtained by certain idealizations, are not, so to say, very sacrosanct any more. A direct study of models of the system on computing machines may possess a heuristic value, at least. A great number of problems were treated in this fashion towards the end of the war and in following years by von Neumann and the writer. At 
first, the probabilistic interpretation was immediately suggested by the physical situation itself. Later, problems of the third class mentioned above were studied. A theory of such mathematical models is still very incomplete. In particular, estimates of fluctuations and accuracy are not as yet developed. Here again, von Neumann contributed a large number of ingenious ways, for example by playing suitable games, of producing sequences of numbers in the given probability distributions. He also devised probabilistic models for treatment of the Boltzmann equation and important stochastic models for some strictly deterministic problems in hydrodynamics. Much of this work is scattered throughout various laboratory reports or is still in manuscript. One certainly hopes that in the near future, an organized selection will be available to the mathematical public.

Theory of automata, probabilistic logic. An account of this work is given in Professor Shannon's article, Von Neumann's contributions to automata theory. This work, like that in game theory, has stimulated, during the last few years, a wide and increasingly expanding number of studies and seems to me to rank with his most fertile ideas. Here a combination of his interest in mathematical logic, computing machines, mathematical analysis, and the knowledge of problems of mathematical physics, come to bear fruit in new constructions. The ideas of Turing, McCulloch, and Pitts on the representation of logical propositions by electrical networks or idealized nervous systems inspired him to propose and outline a general theory of automata. Its notions and terminology come from several fieldsmathematics, electrical engineering, and neurology. Such studies now promise more conquests of mathematics in its ability to formalize, perhaps at first on an extremely simplified level, the workings of an organism and of the nervous system itself.

Nuclear energy, work at Los Alamos. The discovery of the phenomenon of fission in uranium caused by absorption of neutrons with a consequent release of more neutrons came just before the outbreak of the Second World War. A number of physicists realized at once the possibility of a vast release of energy in an exponential reaction in a mass of uranium, and discussions started on quantitative evaluation of arrangements which would lead to utilization of this new source of energy.

Theoretical physicists form a much smaller and more closely knit group than mathematicians and, in general, the interchange of results and ideas is more rapid among them. Von Neumann, whose work in foundations of quantum theory brought him early into contact with most of the leading physicists, was aware of the new experi- 
mental facts and participated, from the beginning, in their speculations on the enormous technological possibilities latent in the phenomena of fission. The outbreak of war found him already engaged in scientific work connected with problems of defense. It was not until late in 1943, however, that he was asked by Oppenheimer to visit the Los Alamos Laboratory as a consultant and began to participate in the work which was to culminate in the construction of the atomic bomb.

As is now well known, the first self-sustaining nuclear chain reaction was established by a group of physicists headed by Fermi in Chicago on December 2, 1942, through the construction of a pile, an arrangement of uranium and a moderating substance where the neutrons are slowed down in order to increase their probability of causing further fissions. A pile forms a very large object and the time for the $e$-folding of the number of neutrons is relatively long. The project established at Los Alamos had as its aim to produce a very fast reaction in a relatively small amount of the 235 isotope of uranium or plutonium, leading to an explosive release of a vast amount of energy. The scientific group began to assemble in late spring of 1943 and by fall of that year a great number of eminent theoretical and experimental physicists were settled there. When von Neumann arrived in Los Alamos, diverse methods of assembling a critical mass of fissionable material were being examined; no scheme was a priori certain of success, one of the problems being whether a sufficiently fast assembly is possible before the nuclear reaction would lead to a mild or mediocre explosion preventing the utilization of most of the material.

E. Teller remembers how Johnny arrived in Lamy (the railroad station nearest Los Alamos), was brought up to the "hill," surrounded at that time by great secrecy, in an official car:

"When he arrived, the Coordinating Council was just in session. Our Director, Oppenheimer, was reporting on the Ottawa meeting in Canada. His speech contained lots of references to most important people and equally important decisions, one of which affected us closely: We could expect the arrival of the British contingent in the near future. After he finished the speech he asked whether there were any questions or comments. The audience was impressed and no questions were asked. Then Oppenheimer suggested that there might be questions on some other topics. After a second or two a deep voice (whose source has been lost to history) spoke, 'When shall we have a shoemaker on the Hill?' Even though no scientific problem was discussed with Johnny at that time, he asserted that as of that moment he was fully familiar with the nature of Los Alamos."

The atmosphere of work was extremely intense at that time and more characteristic of university seminars than technological or engineering laboratories by its informality and the exploratory and, one 
might say, abstract character of scientific discussions. I remember rather vividly that it was with some astonishment that I found, upon arriving at Los Alamos, a milieu reminiscent of a group of mathematicians discussing their abstract speculations rather than of engineers working on a well defined practical project-discussions were going on informally often until late at night. Scientifically, a striking feature of the situation was the diversity of problems, each equally important for the success of the project. For example, there was the problem of the distribution, in space and time, of the neutrons whose number increases exponentially; equally important was the problem of following the increasing deposition of energy by fissions in the material of the bomb, the calculation of hydrodynamical motions in the explosion, the distribution of energy in the form of radiation, and finally, following the course of the motions of the material surrounding the bomb after it has lost its criticality. It was vital to understand all these questions which involved very different mathematical problems.

It is impossible to detail here the contributions of von Neumann; I shall try to indicate some of the more important ones. Early in 1944 a method of implosion was considered for the assembly of the fissionable material. This involves a spherical impulse given to the material, followed by the compression. Von Neumann, Bethe, and Teller were the first to recognize the advantages of this scheme. Teller told him about the experimental work of Neddermeyer and collaborated with von Neumann on working out the essential consequences of such spherical geometry. Von Neumann came to the conclusion that one could produce exceedingly great pressures by this method and it became clear in the discussion that great pressures would bring about considerable compressions as well. In order to start the implosion in a sufficiently symmetrical manner, the original push given by high explosives had to be delivered by simultaneously detonating it from many points. Tuck and von Neumann suggested that it be supplemented by the use of high explosive lenses.

We mentioned before von Neumann's ability, perhaps somewhat rare among mathematicians, to commune with the physicists, understand their language, and to transform it almost instantly into a mathematician's schemes and expressions. Then, after following the problems as such, he could translate them back into expressions in common use among physicists.

The first attempts to calculate the motions resulting from an implosion were extremely schematic. The equations of state of the materials involved were only imperfectly known, but even with crude 
mathematical approximations one was led to equations whose solution was beyond the scope of explicit analytical methods. It became obvious that extensive and tedious numerical work was necessary in order to obtain quantitatively correct results and it is in this connection that computing machines appeared as a necessary aid.

A still more complicated problem is that of the calculation of the characteristics of the nuclear explosion. The amount of energy liberated in it depends on the history of the outward motions which are, of course, governed by the rate of energy deposition and by the thermodynamic properties of the material and radiation at the very high temperatures which are generated. One had to be satisfied for the first experiment with approximate calculations; however, as mentioned before, even the order of magnitude is not easy to estimate without intricate computations. After the end of the war the desire to economize on the material and to maximize its utilization prompted the need for much more precise calculations. Here again von Neumann's contributions to the mathematical treatment of the resulting physical questions were considerable.

Already during the war, the possibilities of thermonuclear reactions were considered, at first only in discussions, then in preliminary calculations. Von Neumann participated actively as a member of an imaginative group which considered various schemes for making possible such reactions on a large scale. The problems involved in treating the conditions necessary for such a reaction and in following its course are even more complex mathematically than those attending a fission explosion (whose characteristics are indeed a prerequisite for following the larger problem). After one discussion in which we outlined the course of such a calculation, von Neumann turned to me and said, "Probably in its execution we shall have to perform more elementary arithmetical steps than the total in all the computations performed by the human race heretofore." We noticed, however, that the total number of multiplications made by the school children of the world in the course of a few years sensibly exceeded that of our problem!

Limitations of space make it impossible to give an account of the innumerable smaller technical contributions of von Neumann welcomed by physicists and engineers engaged in this project.

Von Neumann was very adept in performing dimensional estimates and algebraical and numerical computations in his head without using a pencil and paper. This ability, perhaps somewhat akin to the talent of playing chess blindfolded, often impressed physicists. My impression was that von Neumann did not visualize the physical ob- 
jects under consideration but rather treated their properties as logical consequences of the fundamental physical assumptions; but he was able to play a deductive game with these astonishingly well!

One trait of his scientific personality, which made him very much liked and sought after by those engaged in applications of mathematical techniques, was a willingness to listen attentively even to questions sometimes without much scientific import, but presenting the combinatorial attractions of a puzzle. Many of his interlocutors were helped actively or else consoled by knowing that there is no magic in mathematics known to anyone containing easy answers to their problems. His unselfish willingness to be involved in perhaps too diverse and certainly too numerous activities where mathematical insight might be useful (they are so increasingly common in technology nowadays) put severe demands on his time. In the years following the end of the Second World War, he found himself torn between conflicting demands on his time almost every moment.

Von Neumann strongly believed that the technological revolution initiated by the release of nuclear energy would cause more profound changes in human society, in particular in the development of science, than any technological discovery made in the previous history of the race. In one of the very few instances of talking about his own lucky guesses, he told me that, as a very young man, he believed that nuclear energy would be made available and change the order of human activities during his lifetime!

He participated actively in the early speculations and deliberations on the possibility of controlled thermonuclear reactions. When in 1954 he became a member of the Atomic Energy Commission, he worked on the technical and economical problems relating to the building and operation of fission reactors. In this position he also spent a great deal of time in the organization of studies of mathematical computing machines and the means to make them available to universities and other research centers.

This fragmentary account of von Neumann's diverse achievements and this cursory peregrination through the mathematical disciplines in which he left so many permanent imprints, may raise the question whether there was a thread of continuity throughout his work.

As Poincaré has phrased it: "Il y a des problèmes qu'on se pose et des problèmes qui se posent." Now, fifty years after the great French mathematician formulated this indefinite distinction, the state of mathematics presents this division in a more acute form. Many more of the objects considered by mathematicians are their own free crea- 
tions, of ten, so to say, special generalizations of previous constructions. These are sometimes originally inspired by the schemata of physics, others evolve genetically from free mathematical creationsin some cases prophetically anticipating the actual patterns of physical relations. Von Neumann's thought was obviously influenced by both tendencies. It was his desire to preserve, so far as possible, the connection between the pyramiding mathematical constructions and the increasing combinatorial complexity presented by physics and the natural sciences in general, a connection which seems to be growing more and more elusive.

Some of the great mathematicians of the eighteenth century, in particular Euler, succeeded in incorporating into the domain of mathematical analysis descriptions of many natural phenomena. Von Neumann's work attempted to cast in a similar role the mathematics stemming from set theory and modern algebra. This is of course, nowadays, a vastly more difficult undertaking. The infinitesimal calculus and the subsequent growth of analysis through most of the nineteenth century led to hopes of not merely cataloguing, but of understanding the contents of the Pandora's box opened by the discoveries of physical sciences. Such hopes are now illusory, if only because the real number system of the Euclidean space can no longer claim, algebraically, or even only topologically, to be the unique or even the best mathematical substratum for physical theories. The physical ideas of the 19th century, dominated mathematically by differential and integral equations and the theory of analytic functions, have become inadequate. The new quantum theory requires on the analytic side a set-theoretically more general point of view, the primitive notions themselves involving probability distributions and infinite-dimensional function spaces. The algebraical counterpart to this involves a study of combinatorial and algebraic structures more general than those presented by real or complex numbers alone. Von Neumann's work came at a time when the whole complex of ideas stemming from Cantor's set theory and the algebraical work of Hilbert, Weyl, Noether, Artin, Brauer, and others could be exploited for this purpose.

Another major source from which general mathematical investigations are beginning to develop is a new kind of combinatorial analysis stimulated by the recent fundamental researches in the biological sciences. Here, the lack of general method at the present time is even more noticeable. The problems are essentially non-linear, and of an extremely complex combinatorial character; it seems that many years of experimentation and heuristic studies will be necessary be- 
fore one can hope to achieve the insight required for decisive syntheses. An awareness of this is what prompted von Neumann to devote so much of his work of the last ten years to the study and the construction of computing machines and to formulate a preliminary outline for the study of automata.

Surveying von Neumann's work and seeing how ramified and extended it is, one could say with Hilbert: "One is led to ask oneself whether the science of mathematics will not end, as has been the case for a long time now for other sciences, in a subdivision of separate parts whose representatives will barely understand each other and whose connections will continue to diminish? I neither think so nor hope for this; the science of mathematics is an indivisible whole, an organism whose vital force has as its premise the indissolubility of its parts. Whatever the diversity of subjects of our science in its details, we are nonetheless struck by the equivalence of the logical procedures, the relation of ideas in the whole of science and the numerous analogies in its different domains ...."14 Von Neumann's work was a contribution to this ideal of the universality and organic unity of mathematics.

Among the numerous scientific positions held by von Neumann, one should name his Gibbs Lectureship in the American Mathematical Society (1947); he gave the American Mathematical Society Colloquium Lecture in 1937 and was Vanuxem Lecturer at Princeton University in 1953. He was president of the American Mathematical Society from 1951-1953. During his years as a professor at the Institute in Princeton, he gave lectures, too numerous to list, at various learned societies and academic institutions.

He served as a co-editor of the Annals of Mathematics in Princeton from 1933-1957, and of Compositio Mathematica (Amsterdam, Netherlands) from 1935-1957.

The society memberships included: American Mathematical Society; American Physical Society; Econometric Society; International Statistical Institute, The Hague, Netherlands; Sigma Xi.

He was a member of the following academies:

Academia Nacional de Ciencias Exactas, Lima, Peru;

Academia Nazionale dei Lincei, Rome, Italy;

American Academy of Arts and Sciences;

American Philosophical Society;

Instituto Lombardo di Scienze e Lettere, Milano, Italy;

14 Hilbert: Problèmes futurs des Mathématiques, Comptes-Rendus, 2ème Congrès International de Mathématiques, Paris, 1900. 
National Academy of Sciences;

Royal Netherlands Academy of Sciences and Letters, Amsterdam, Netherlands.

He was awarded the following Honorary Doctors degrees: Princeton University, 1947; University of Pennsylvania and Harvard University, 1950; University of Istanbul, Turkey, and University of Maryland, 1950, also Columbia University and the Technische Hochschule in Munich.

Among the distinctions and honors received:

Rockefeller Fellowship-1926;

Bôcher Prize, American Mathematical Society-1937;

Medal for Merit (Presidential Award), distinguished Civilian

Service Award, U. S. Navy-1947;

Medal of Freedom (Presidential Award)-1956;

Albert Einstein Commemorative Award-1956:

Enrico Fermi Award-1956.

An incomplete list of scientific and organizational activities contains the following positions: From 1940-1957, he was a member of the Scientific Advisory Committee, Ballistic Research Laboratories, Aberdeen Proving Ground, Maryland; the Navy Bureau of Ordnance, Washington, D. C. from 1941-1955; consultant to Los Alamos Scientific Laboratory 1943-1955; also the Naval Ordnance Laboratory, Silver Spring, Maryland from 1947-1955; member of the Research and Development Board, Washington, D. C. 1949-1953; a consultant to the Oak Ridge National Laboratory, Oak Ridge, Tennessee 19491954; member from 1950 to 1955 of the Armed Forces Special Weapons Project, Washington, D. C.; also in Washington a member of the Scientific Advisory Board, U. S. Air Force, Washington, D. C. 1951-1957; a member of the General Advisory Committee by presidential appointment 1952-1954; and on the Technical Advisory Panel on Atomic Energy, Washington, D. C. 1953-1957; Chairman of the Advisory Committee on Guided Missiles (1954-1957 with Clark Millikan as acting chairman in 1956).

\section{APPENDIX 1}

\section{BIBLIOGRAPHY}

1922 1. Über die Lage der Nullstellen gewisser Minimumpolynome. With M. Fekete. Jber. Deutschen Math. Verein. vol. 31 (1922) pp. 125-138.

1923 2. Zur Einführung der transfiniten Ordnungszahlen, Acta Univ. Szeged vol. 1 (1923) pp. 199-208.

1925 3. Eine Axiomatisierung der Mengenlehre, J. Reine Angew. Math. vol. 154 (1925) pp. 219-240. 
4. Egyenletesen silril szamsorozatok, Math. Phys. Lapok vol. 32 (1925) pp. $32-40$.

5. Zur Prifferschen Theorie der idealen Zahlen, Acta Univ. Szeged vol. 2 (1926) pp. 193-227.

6. Az altalanos Nalmazelmelet axiomatikus folépitése (Doctor's thesis, Univ. of Budapest.) Cf. [18] (1926).

1927 7. Uber die Grundlagen der Quantenmechanik. With D. Hilbert and L. Nordheim. Math. Ann. vol. 98 (1927) pp. 1-30.

8. Zur Theorie der Darstellungen kontinuierlicher Gruppen, Preuss. Akad. Wiss. Sitzungsber. (1927) pp. 76-90.

9. Mathematische Begrundung der Quantenmechanik, Nachr. Ges. Wiss. Göttingen (1927), pp. 1-57.

10. Wahrscheinlichkeitstheoretischer Aufbau der Quantenmechanik, Nachr. Ges. Wiss. Gottingen (1927) pp. 245-272.

11. Thermodynamik quantenmechanischer Gesamtheiten, Nachr. Ges. Wiss. Göttingen (1927) pp. 273-291.

12. Zur Hilbertschen Beweistheorie, Math. Zeit. vol. 26 (1927) pp. 1-46.

1928 13. Allgemeine Eigenwerttheorie symmetrischer Funktionaloperatoren, "Habilitations-schrift," Univ. of Berlin, 1928, Cf. [30].

14. Zerlegung des Intervalles in abzdhlbar viele kongruente Teilmengen, Fund. Math. vol. 11 (1928) pp. 230-238.

15. Ein System algebraisch unabhängiger Zahlen, Math. Ann. vol. 99 (1928) pp. 134-141.

16. Über die Definition durch transfinite Induktion, und verwandte Fragen der allgemeinen Mengenlehre, Math. Ann. vol. 99 (1928) pp. 373-391.

17. Zur Theorie der Gesellschaftsspiele, Math. Ann. vol. 100 (1928) pp. 295320.

18. Die Axiomatisierung der Mengenlehre, Math. Zeit. vol. 27 (1928) pp. 669-752.

19. Zur Erklärung einiger Eigenschaften der Spektren aus der Quantenmechanik des Drehelektrons, I. With E. Wigner. Zschr. f. Phys. vol. 47 (1928) pp. 203-220.

20. Einige Bemerkungen zur Diracschen Theorie des Drehelektrons, Zschr. f. Phys. vol. 48 (1928) pp. 868-881.

21. Zur Erklärung einiger Eigenschaften der Spektren aus der Quantenmechanik des Drehelektrons, II. With E. Wigner. Zschr. f. Phys. vol. 49 (1928) pp. 73-94. Cf. [19].

22. Zur Erklärung einiger Eigenschaften der Spektren aus der Quantenmechanik des Drehelektrons, III. With E. Wigner. Zschr. f. Phys. vol. 51 (1928) pp. 844-858. Cf. [19].

23. Über eine Widerspruchfreiheitsfrage der axiomatischen Mengenlehre, J. Reine Angew. Math. vol. 160 (1929) pp. 227-241.

24. Über die analytischen Eigenschaften von Gruppen linearer Transformationen und ihrer Darstellungen, Math. Zeit. vol. 30 (1929) pp. 3-42.

25. Über merkwirdige diskrete Eigenwerte. With E. Wigner. Phys. Zschr. vol. 30 (1929) pp. 465-467.

26. Über das Verhalten von Eigenwerten bei adiabatischen Prozessen. With E. Wigner. Phys. Zschr. vol. 30 (1929) pp. 467-470.

27. Beweis des Ergodensatzes und des $H$-Theorems in der neuen Mechanik, Zschr. f. Phys. vol. 57 (1929) pp. 30-70. 
28. Zur allgemeinen Theorie des Masses, Fund. Math. vol. 13 (1929) pp. 73116.

29. Zusatz zur Arbeit "Zur allgemeinen ... " Fund. Math. vol. 13 (1929) p. 333. Cf. [28].

30. Allgemeine Eigenwerttheorie Hermitescher Funktionaloperatoren. Math. Ann. vol. 102 (1929) pp. 49-131.

31. Zur Algebra der Funktionaloperatoren und Theorie der normalen Operatoren, Math. Ann. vol. 102 (1929) pp. 370-427.

32. Zur Theorie der unbeschränkten Matrizen, J. Reine Angew Math. vol. 161 (1929) pp. 208-236.

1930 33. Über einen Hilfssatz der Variationsrechnung, Abh. Math. Sem. Hansischen Univ. vol. 8 (1930) pp. 28-31.

1931 34. Über Funktionen von Funktionaloperatoren, Ann. Math. vol. 32 (1931) pp. 191-226.

35. Algebraische Repräsentanten der Funktionen "bis auf eine Menge vom Maasse Null," J. Reine Angew. Math. vol. 161 (1931) pp. 109-115.

36. Die Eindeutigkeit der Schrödingerschen Operatoren, Math. Ann. vol. 104 (1931) pp. 570-578.

37. Bemerkungen zu den Ausfïhrungen von Herrn St. Lesniewski über meine Arbeit "Zur Hilbertschen Beweistheorie," Fund. Math. vol. 17 (1931) pp. 331-334.

38. Die formalistische Grundlegung der Mathematik. Report to the Congress, Königsberg, Sept. 1931. Erkenntniss, 2, pp. 116-121.

1932 39. Zum Beweise des Minkowskischen Satzes über Linearformen, Math. Zeit. vol. 30 (1932) pp. 1-2.

40. Über adjungierte Funktionaloperatoren, Ann. of Math. vol. 33 (1932) pp. 294-310.

41. Proof of the quasi-ergodic hypothesis, Proc. Nat. Acad. Sci. U.S.A. vol. 18 (1932) pp. 70-82.

42. Physical applications of the quasi-ergodic hypothesis, Proc. Nat. Acad. Sci. U.S.A. vol. 18 (1932) pp. 263-266.

43. Dynamical systems of continuous spectra. With B. O. Koopman. Proc. Nat. Acad. Sci. U.S.A. vol. 18 (1932) pp. 255-263.

44. Über einen Satz von Herrn M. H. Stone, Ann. of Math. vol. 33 (1932) pp. 567-573.

45. Einige Sätze uber messbare Abbildungen, Ann. of Math. vol. 33 (1932) pp. 574-586.

46. Zur Operatorenmethode in der klassischen Mechanik, Ann. of Math. vol. 33 (1932) pp. 587-642.

47. Zusatze zur Arbeit "Zur Operatorenmethode... ", Ann. of Math. vol. 33 (1932) pp. 789-791. Cf. [46].

47a. Mathematische Grundlagen der Quantenmechanik, J. Springer, Berlin, 1932; Dover Publications, New York, 1943; Presses Universitaires de France, 1947; Instituto de Mathematicas "Jorge Juan," Madrid, 1949; Translation from German ed. by Robert T. Beyer, Princeton University Press, 1955.

1933 48. Die Einführung analytischer Parameter in topologischen Gruppen, Ann. of Math. vol. 34 (1933) pp. 170-190.

49. A koordinâta-mérés pontosságanak hatârai az elektron Dirac-féle elméletében (Über die Grenzen der Koordinatenmessungs-Genauigkeit in der 
Diracschen Theorie des Elektrons), Mat. es Termeszettud ... Ertesito vol. 50 (1933) pp. 366-385.

1934 50. On an algebraic generalization of the quantum mechanical formalism. With P. Jordan and E. Wigner. Ann. of Math. vol. 35 (1934) pp. 29-64.

51. Zum Haarschen Maass in topologischen Gruppen, Compositio Math. vol. 1 (1934) pp. 106-114.

52. Almost periodic functions in a group. I, Trans. Amer. Math. Soc. vol. 36 (1934) pp. 445-492.

53. The Dirac equation in projective relativity. With $\mathrm{A}$. $\mathrm{H}$. Taub and $\mathrm{O}$. Veblen. Proc. Nat. Acad. Sci. U.S.A. vol. 20 (1934) pp. 383-388.

1935 54. On complete topological spaces, Trans. Amer. Math. Soc. vol. 37 (1935) pp. 1-20.

55. Almost periodic functions in groups. II. With S. Bochner. Trans. Amer. Math. Soc. vol. 37 (1935) pp. 21-50.

56. On compact solutions of operational-differential equations. I. With S. Bochner. Ann. of Math. vol. 36 (1935) pp. 255-291.

57. Charakterisierung des Spektrums eines Integraloperators. Actualités Scientifiques et Industrielles Series, 229. Exposés Math. publiés à la mémoire de J. Herbrand, no. 13, Paris, 1935, 20 pp.

58. On normal operators. Proc. Nat. Acad. Sci. U.S.A. vol. 21 (1935) pp. 366369.

59. On inner products in linear, metric spaces. With P. Jordan. Ann. of Math. vol. 36 (1935) pp. 719-723.

60. The determination of representative elements in the residual classes of a Boolean algebra. With M. H. Stone. Fund. Math. vol. 25 (1935) pp. 353-378.

1936 61. On a certain topology for rings of operators, Ann. of Math. vol. 37 (1936) pp. 111-115.

62. On rings of operators. With F. J. Murray. Ann. of Math. vol. 37 (1936) pp. 116-229.

63. On an algebraic generalization of the quantum mechanical formalism (Part I). Rec. Math (Mat. Sbornik) N. S. vol. 1 (1936) pp. 415-484.

64. The uniqueness of Haar's measure, Rec. Math (Mat. Sbornik) N. S. vol. 1 (1936) pp. 721-734.

65. The logic of quantum mechanics. With G. Birkhoff. Ann. of Math. vol. 37 (1936) pp. 823-843.

66. Continuous geometry, Proc. Nat. Acad. Sci. U.S.A. vol. 22 (1936) pp. 92-100.

67. Examples of continuous geometries, Proc. Nat. Acad. Sci. U.S.A. vol. 22 (1936) pp. 101-108.

68. On regular rings. Proc. Nat. Acad. Sci. U.S.A. vol. 22 (1936) pp. 707713.

1937 69. On some analytic sets defined by transfinite induction. With C. Kuratowski. Ann. of Math. vol. 38 (1937) pp. 521-525.

70. On rings of operators, II. With F. J. Murray. Trans. Amer. Math. Soc. vol. 41 (1937) pp. 208-248.

71. Some matrix-inequalities and metrization of matrix-space. Tomck. Univ. Rev. vol. 1 (1937) pp. 286-300.

72. Über ein ökonomisches Gleichungssystem und eine Verallgemeinerung des 
Brouwerschen Fixpunktsatzes, Erg. eines Math. Coll., Vienna, edited by K. Menger, vol. 8, 1937, pp. 73-83.

73. Algebraic theory of continuous geometries, Proc. Nat. Acad. Sci. U.S.A. vol. 23 (1937) pp. 16-22.

74. Continuous rings and their arithmetics, Proc. Nat. Acad. Sci. U.S.A. vol. 23 (1937) pp. 341-349.

1938 75. On infinite direct products, Compositio Math. vol. 6 (1938) pp. 1-77.

1940 76. On the transitivity of perspective mappings. With I. Halperin. Ann. of Math. vol. 41 (1940) pp. 87-93.

77. On rings of operators, III, Ann. of Math. vol. 41 (1940) pp. 94-161.

78. Minimally almost periodic groups. With E. Wigner. Ann. of Math. vol. 41 (1940) pp. 746-750.

78a. The estimation of the probable error from succesive differences. With R. H. Kent. Aberdeen Proving Ground, Md., Report No. 175, 1940, 19 pp.

1941 79. The mean square successive difference. With $R$. $H$. Kent, $H$. R. Bellinson and B. I. Hart. Ann. Math. Statist. vol. 12 (1941) pp. 153-162.

80. Fourier integrals and metric geometry. With I. J. Schoenberg. Trans. Amer. Math. Soc. vol. 50 (1941) pp. 226-251.

81. Distribution of the ratio of the mean square successive difference to the variance, Ann. Math. Statist. vol. 12 (1941) pp. 367-395.

1942 82. A further remark concerning the distribution of the ratio of the mean square successive difference to the variance, Ann. Math. Statist. vol. 13 (1942) pp. 86-88.

83. Operator methods in classical mechanics, II. With P. R. Halmos. Ann. of Math. vol. 43 (1942) pp. 332-350.

84. The statistics of the gravitational field arising from a random distribution of stars, I. With S. Chandrasekhar. The Astrophysical Journal vol. 95 (1942) pp. 489-531.

85. Tabulation of the probabilities for the ratio of the mean square successive difference to the variance. By B. I. Hart, with a note by J. von Neumann. Ann. Math. Statist. vol. 13 (1942) pp. 207-214.

86. Approximative properties of matrices of high finite order, Portugaliae Mathematica vol. 3 (1942) pp. 1-62.

1943 87. On rings of operators, IV. With F. J. Murray. Ann. of Math. vol. 44 (1943) pp. 716-808.

88. The statistics of the gravitational field arising from a random distribution of stars. II. The speed of fluctuations; dynamical friction; spatial correlations. With S. Chandrasekhar. The Astrophysical Journal vol. 97 (1943) pp. 1-27.

89. On some algebraical properties of operator rings, Ann. of Math. vol. 44 (1943) pp. 709-715.

1944 90. Theory of games and economic behavior. With O. Morgenstern. Princeton University Press $(1944,1947,1953) 625$ pp.

1945 90a. A model of general economic equilibrium, Rev. Economic Studies, vol. 13 (1), (1945-1946) pp. 1-9.

1946 91. Solution of linear systems of high order. With V. Bargmann and D. Montgomery. Report prepared for Navy BuOrd under Contract Nord-959625 , Oct. 1946, 85 pp.

92. Preliminary discussion of the logical design of an electronic computing instrument. Part I, Vol. I. With A. W. Burks and H. H. Goldstine. Re- 
port prepared for U. S. Army Ord. Dept. under Contract W-36-034ORD-7481 (28 June 1946, 2d ed. September 2, 1947), 42 pp.

92a. The cross-space of linear transformations. II. With R. Schatten. Ann. of Math. vol. 47 (1946) p. 608.

92b. The cross-space of linear transformations. III. With R. Schatten. Ann. of Math. vol. 49 (1948) p. 557.

93. The Mathematician. "The Works of the Mind." U. of Chicago Press, 1947, pp. 180-196.

94. Numerical inverting of matrices of high order. With H. H. Goldstine. Bull. Amer. Math. Soc. vol. 53 (1947) pp. 1021-1099.

95. Planning and coding of problems for an electronic computing instrument. Part II, Vol. I. With H. H. Goldstine. Report prepared for U. S. Army Ord. Dept. under Contract W-36-034-ORD-7481, 1947, 69 pp.

96. Planning and coding of problems for an electronic computing instrument. Part II, Vol. II. With H. H. Goldstine. Report prepared for U. S. Army Ord. Dept. under Contract W-36-034-ORD-7481, 1948, 68 pp.

97. Planning and coding of problems for an electronic computing instrument. Part II, Vol. III. With H. H. Goldstine. Report prepared for U. S. Army Ord. Dept. under Contract W-36-034-ORD-7481, 1948, 23 pp.

98. On the theory of stationary detonation waves, File No. X122, September 20, 1948, BRL, Aberdeen Proving Ground, Md., 26 pp.

1949 99. On rings of operators. Reduction theory, Ann. of Math. vol. 50 (1949) pp. 401-485.

1950 100. A method for the numerical calculation of hydrodynamic shocks. With R. D. Richtmyer. Journal of Applied Physics vol. 21 (1950) pp. 232-237.

101. Functional Operators-Vol. I: Measures and Integrals, Ann. of Math. Studies, no. 21, 261 pp.; Vol. II: The geometry of orthogonal spaces, Ann. of Math. Studies, no. 22, 107 pp., Princeton University Press, 1950.

102. Solutions of games by differential equations. With G. W. Brown, "Contributions to the Theory of Games," Ann. of Math. Studies, no. 24, Princeton University Press, 1950, pp. 73-79.

103. Statistical treatment of values of first 2000 decimal digits of $e$ and of $P i$ calculated on the ENIAC. With N. C. Metropolis and G. Reitwiesner. Mathematical Tables and Other Aids to Computation vol. 4 (1940) pp. 109-111.

104. Numerical integration of the barotropic vorticity equation. With J. G. Charney and R. Fjortoft. Tellus 2 (1950) pp. 237-254.

105. A theorem on unitary representations of semisimple lie groups. With I. E. Segal. Ann. of Math. vol. 52 (1950) pp. 509-517.

106. Eine Spektraltheorie für allgemeine Operatoren eines unitären Raumes. Nachr. Ges. Wiss. Göttingen vol. 4 (1950-51) pp. 258-281.

107. The future of high-speed computing, Proc., Camp. Sem., Dec. 1949, published and copyrighted by IBM, 1951, p. 13.

108. Discussion of the existence and uniqueness or multiplicity of solutions of the aerodynamical equations (Chapter 10) of the Problems of Cosmical Aerodynamics, Proceedings of the Symposium on the Motion of Gaseous Masses of Cosmical Dimensions held at Paris, August 16-19, 1949. Central Air Doc. Office, 1951, pp. 75-84.

109. Numerical inverting of matrices of high order, II. With $\mathrm{H}$. H. Goldstine. Proc. Amer. Math. Soc. vol. 2 (1951) pp. 188-202.

110. Various techniques used in connection with random digits (Chap. 13) of 
proceedings of symposium on "Monte Carlo Method" held June-July 1949 in Los Angeles. Nat'l. BuStandards, Applied Math. Series 12, June 11, 1951, pp. 36-38.

111. The general and logical theory of automata. "Cerebral Mechanisms in Behavior-The Hixon Symposium," September 1948, Pasadena, edited by L. A. Jeffress. John Wiley and Sons, Inc., New York, 1951, pp. 1-31.

1952 112. Five notes on continuous geometry, Prepared by W. Givens for use of participants in seminar at Univ. of Tenn., summer 1952. Includes nos. $66,67,68,73,74$.

1953 113. A certain zero-sum two-person game equivalent to the optimal assignment problem. "Contributions to the Theory of Games," Vol. II, Ann. of Math. Studies, no. 28, Princeton University Press, 1953, pp. 5-12.

114. Two variants of poker. With D. G. Gillies and J. P. Mayberry. "Contributions to the Theory of Games," Vol. II. Ann. of Math. Studies, no. 28, Princeton University Press 1953, pp. 13-50.

1954 115. Significance of Loewner's theorem in the quantum theory of collisions. With E. P. Wigner. Ann. of Math. vol. 59 (1954) pp. 418-433.

116. The role of mathematics in the sciences and in society. Address before Assoc. of Princeton Graduate Alumni, June 16, 1954.

117. A numerical method to determine optimum strategy, Naval Res. Logistics Quarterly, vol. 1, no. 2, June, 1954, pp. 109-115.

118. The NORC and problems in high speed computing. Speech at first public showing of IBM Naval Ordnance Research Calculator, December 2, 1954.

119. Entwicklung und Ausnutzung neuerer mathematischer Maschinen. Arbeitsgemeinschaft für Forschung des Landes Nordrhein-Westfalen, Heft 45. Düsseldorf, September 15, 1954.

1955 120. Can we survive technology?, Fortune, June, 1955.

121. On the permutability of self-adjoint operators. With A. Devinatz and A. E. Nussbaum. Ann. of Math. vol. 62 (1955) pp. 199-203.

122. Continued fraction expansion of $2^{1 / 3}$. With Bryant Tuckerman. Mathematical Tables and Other Aids to Computation, IX, no. 49, January, 1955.

123. Blast wave calculation. With $\mathrm{H}$. $\mathrm{H}$. Goldstine. Communications on Pure and Applied Mathematics. vol. VIII (1955) pp. 327-353.

1956 124. Probabilistic logics and the synthesis of reliable organisms from unreliable components. "Automata Studies," edited by C. E. Shannon and J. McCarthy, Princeton University Press, 1956, pp. 43-98.

125. Impact of atomic energy on the physical and chemical sciences. Speech at M.I.T. Alumni Day Symposium. Tech. Rev., Nov. 1955, pp. 15-17.

\section{APPENDIX 2}

\section{Abstracts of papers PRESENTEd to THE American Mathematical Society}

1. J. von Neumann, Almost periodic functions in a group. I, Bull. Amer. Math. Soc. vol. 40 (1934) p. 224.

2. - On complete topological spaces, Bull. Amer. Math. Soc. vol. 41 (1935) p. 35.

3. S. Bochner and J. von Neumann, Almost periodic functions in groups. II, Bull. Amer. Math. Soc. vol. 41 (1935) p. 35. 
4. J. von Neumann, Representations and ray-representations in quantum mechanics, Bull. Amer. Math. Soc. vol. 41 (1935) p. 305.

5. - On the uniqueness of invariant Lebesgue measures, Bull. Amer. Math. Soc. vol. 42 (1936) p. 343.

6. I. J. Schoenberg and J. von Neumann, Fourier integrals and metric geometry, Bull. Amer. Math. Soc. vol. 42 (1936) p. 632.

7. F. J. Murray and J. von Neumann, On rings of operators. II, Bull. Amer. Math. Soc. vol. 42 (1936) p. 808.

8. I. Halperin and J. von Neumann, On the transitivity of perspective mappings in complemented modular lattices, Bull. Amer. Math. Soc. vol. 43 (1937) p. 37.

9. I. J. Schoenberg and J. von Neumann, Fourier integrals and metric geometry, II, Bull. Amer. Math. Soc. vol. 45 (1939) p. 79.

10. P. R. Halmos and J. von Neumann, Operator methods in classical mechanics, II, Bull. Amer. Math. Soc. vol. 47 (1941) p. 696.

11. S. M. Ulam and J. von Neumann, Random ergodic theorems, Bull. Amer. Math. soc. vol. 51 (1945) p. 660.

12. R. Schatten and J. von Neumann, The cross-space of linear transformations, II, Bull. Amer. Math. Soc. vol. 52 (1946) p. 67.

13. E. R. Lorch and J. von Neumann, On the Euclidean character of the perpendicularity relation, Bull. Amer. Math. Soc. vol. 53 (1947) p. 489.

14. S. Ulam and J. von Neumann, On the group of homeomorphisms of the surface of the sphere, Bull. Amer. Math. Soc. vol. 53 (1947) p. 506.

15. - On combination of stochastic and deterministic processes, Bull. Amer. Math. Soc. vol. 53 (1947) p. 1120.

16. H. H. Goldstine and J. von Neumann, Some estimates on the numerical stability of the elimination method for inverting matrices of high order, Bull. Amer. Math. Soc. vol. 53 (1947) p. 1123.

17. R. Schatten and J. von Neumann, The cross-space of linear transformations, III, Bull. Amer. Math. Soc. vol. 54 (1948) p. 637.

Los Alamos Scientific Laboratory 